TRANSACTIONS OF THE

AMERICAN MATHEMATICAL SOCIETY

Volume 355, Number 10, Pages 4223-4240

S 0002-9947(03)03329-4

Article electronically published on June 10, 2003

\title{
THE AUTOHOMEOMORPHISM GROUP OF THE ČECH-STONE COMPACTIFICATION OF THE INTEGERS
}

\author{
JURIS STEPRĀNS
}

\begin{abstract}
It is shown to be consistent that there is a nontrivial autohomeomorphism of $\beta \mathbb{N} \backslash \mathbb{N}$, yet all such autohomeomorphisms are trivial on a dense $P$-ideal. Furthermore, the cardinality of the autohomeomorphism group of $\beta \mathbb{N} \backslash \mathbb{N}$ can be any regular cardinal between $2^{\aleph_{0}}$ and $2^{2^{\aleph_{0}}}$. The model used is one due to Velickovic in which, coincidentally, Martin's Axiom also holds.
\end{abstract}

\section{INTRODUCTION}

An automorphism of $\mathcal{P}(\omega) /[\omega]<\aleph_{0}$ _or, equivalently, an autohomeomorphism of $\beta \mathbb{N} \backslash \mathbb{N}$ - is said to be trivial if there is a bijection between co-finite subsets of the integers which induces it; an automorphism is said to be somewhere trivial if its restriction to $\mathcal{P}(A) /[A]^{<\aleph_{0}}$ is trivial for some $A \in[\omega]^{\aleph_{0}}$. It was shown by Shelah, [3. pp. 129-152] that it is consistent with ZFC that all automorphisms of $\mathcal{P}(\omega) /[\omega]<\aleph_{0}$ are trivial; therefore, it is also consistent that the size of the autohomeomorphism group of $\beta \mathbb{N} \backslash \mathbb{N}$ is $2^{\aleph_{0}}$. This is in contrast to most other models of set theory where the size of the autohomeomorphism group of $\beta \mathbb{N} \backslash \mathbb{N}$ is $2^{2^{\aleph_{0}}}$. This might lead one to ask whether there is a dichotomy similar to that for Borel sets, that is, the size of the autohomeomorphism group of $\beta \mathbb{N} \backslash \mathbb{N}$ is either $2^{\aleph_{0}}$ or $2^{2^{\aleph_{0}}}$. The present paper will show that no such dichotomy exists, as well as providing some detailed information about the nature of automorphisms of $\mathcal{P}(\omega) /[\omega]<\aleph_{0}$ in a certain class of models. In particular, it will be shown that in these models all automorphisms of $\mathcal{P}(\omega) /[\omega]^{<\aleph_{0}}$ are trivial on a dense $P$-ideal. The fact that they are trivial on a dense set was established in 5 .

In order to be more precise the following definitions will be introduced.

Definition 1.1. The relation $\equiv^{*}$ has the standard meaning; namely, $A \equiv{ }^{*} B$ if and only if $|A \Delta B|<\aleph_{0}$ (here, $A \Delta B=(A \backslash B) \cup(B \backslash A)$ ). Also. $A \subseteq^{*} B$ is defined to mean that $|A \backslash B|<\aleph_{0}$. If $A \subseteq \omega$, then the equivalence class of $A$ with respect to $\equiv^{*}$ will be denoted by $[A]$.

The notion of triviality can now be precisely formulated.

Definition 1.2. A homomorphism $\Phi: \mathcal{P}(\omega) /[\omega]^{<\aleph_{0}} \rightarrow \mathcal{P}(\omega) /[\omega]^{<\aleph_{0}}$ is said to be trivial on $A \subseteq \omega$ if there is $A^{\prime} \equiv^{*} A$ and a one-to-one function $f: A^{\prime} \rightarrow \omega$ such that $\Phi([B])=[f(B)]$ for every $B \subseteq A$. The function $f$ is said to induce $\Phi$ on $A$.

Received by the editors January 8, 2001 and, in revised form, March 10, 2003.

2000 Mathematics Subject Classification. Primary 03E35.

Research for this paper was partially supported by NSERC of Canada.

(C)2003 American Mathematical Society 
A homomorphism will be said to be somewhere trivial if there is some $A \in[\omega]^{\aleph_{0}}$ on which it is trivial. A homomorphism is trivial if it is trivial on $\omega$.

It has already been mentioned that it was shown in [3] that it is consistent that all automorphisms of $\mathcal{P}(\omega) /[\omega]^{<\aleph_{0}}$ are trivial. The argument relied on the oracle chain condition, and it was not clear what the effect of Martin's Axiom was on the question. This was partially answered in [4] where it was shown that the Proper Forcing Axiom (PFA) implies that all automorphisms of $\mathcal{P}(\omega) /[\omega]^{<\aleph_{0}}$ are trivial; for related results see 2 . The other half of the answer was provided by Velickovic in 9] where it is shown that it is consistent with Martin's Axiom that a nontrivial automorphism of $\mathcal{P}(\omega) /[\omega]<\aleph_{0}$ exists.

\section{Notation AND BACKGROUND}

The notation used throughout will adhere to accepted convention. If $n$ and $m$ are integers, then $[n, m)$ will denote the interval $m \backslash n$ even though the latter notation is more compact. When a partial order appears in a forcing context, greater conditions will be assumed to force more information unless a natural ordering, such as $\subseteq$ or $\subseteq^{*}$, on the set is used.

Notation 2.1. The notation $i_{X}$ will be used to denote the constant function whose domain is $X$ and which has value $i$ at each point in $X$.

Notation 2.2. Whenever reference is made to a topology on $\mathcal{P}(\omega)$, this will be to the Cantor set topology under the canonical identification of $2^{\omega}$ with $\mathcal{P}(\omega)$; in other words, a natural base for this topology consists of all sets of the form

$$
\left\{A \subseteq \omega: 1_{A} \cup 0_{\omega \backslash A} \supseteq g\right\}
$$

where $g$ is a finite partial function from $\omega$ to 2 .

Notation 2.3. The notation $\stackrel{\circ}{x}$ will be used to indicate names not of the form $\check{x}$ in forcing statements, the assumption being that all symbols occurring in a forcing expression are of the form $\check{x}$ unless explicitly indicated otherwise by ${ }^{\circ}$. For example, in the expression $p \Vdash$ " $n \in \stackrel{\circ}{y}$ " it is being assumed that $n$ is the same as $\check{n}$.

Definition 2.1. If $\Phi$ is an automorphism of $\mathcal{P}(\omega) /[\omega]^{<\aleph_{0}}$, define $\mathcal{T}(\Phi)$ to be the ideal of all $X \in[\omega]^{\aleph_{0}}$ such that $\Phi$ is trivial on $X$.

The following theorem of 9] offers an alternate characterization of triviality which has proven to be very useful.

Lemma 2.1 (Velickovic). If $\Phi: \mathcal{P}(\omega) /[\omega]<\aleph_{0} \rightarrow \mathcal{P}(\omega) /[\omega]<\aleph_{0}$ is an automorphism and there exist Borel functions $\psi_{n}: \mathcal{P}(\omega) \rightarrow \mathcal{P}(\omega)$ for $n \in \omega$ such that for all but a meagre set of $A \in \mathcal{P}(\omega)$ there is $n \in \omega$ such that $\left[\psi_{n}(A)\right]=\Phi([A])$, then $\Phi$ is trivial.

The meagre set of exceptions is not mentioned in [9, but it follows from minor modifications to the argument there.

\section{Automorphisms of $\mathcal{P}(\omega) /[\omega]^{<\aleph_{0}}$ Are trivial on a dense $P$-IDeal}

The arguments to be presented in this section will be a modification and combination of arguments from [3, pp. 129-152] (or [6] pp. 175-193]), [4] and [9]. The 
following partial order $\mathbb{P}$, was introduced by Velickovic in [9], following Baumgartner, to add a nontrivial automorphism of $\mathcal{P}(\omega) /[\omega]^{<\aleph_{0}}$ while doing as little else as possible - at least assuming PFA.

Definition 3.1. The partial order $\mathbb{P}$ is defined to consist of all bijections $f: A \rightarrow B$ where

- $A \subseteq \omega$ and $B \subseteq \omega$;

- for all $i \in A$ and $n \in \omega, 2^{n} \leq f(i)<2^{n+1}$ if and only if $2^{n} \leq i<2^{n+1}$;

- $\lim \sup _{n \rightarrow \omega}\left|\left[2^{n}, 2^{n+1}\right) \backslash A\right|=\omega$ and so, by the previous condition, $\lim \sup _{n \rightarrow \omega}\left|\left[2^{n}, 2^{n+1}\right) \backslash B\right|=\omega$.

The ordering on $\mathbb{P}$ is $\subseteq^{*}$.

The terms $2^{n}$ are not crucial in the definition of $\mathbb{P}$, since any sequence of intervals whose size tends to infinity could equally well have been used. Further modifications to the partial order are also possible (some can be found in [4]) but will not be important in the present context. It is, however, useful to note the following observation from [5].

Lemma 3.1. Assume $M A_{\eta}$ and that $\left\{f_{\xi}\right\}_{\xi \in \eta}$ is an increasing sequence from $\mathbb{P}$. Suppose further that there is $f^{\prime}$ such that $f^{\prime} \supseteq^{*} f_{\xi}$ for each $\xi \in \eta$. Then there is $f \in \mathbb{P}$ such that $f \supseteq^{*} f_{\xi}$ for each $\xi \in \eta$.

Definition 3.2. The countable support product of $\kappa$ copies of $\mathbb{P}$ will be denoted by $\mathbb{P}(\kappa)$; in other words, if $f \in \mathbb{P}(\kappa)$, then $f: \kappa \rightarrow \mathbb{P}$ and $f(\alpha)=\emptyset$ for all but countably many $\alpha$. The operations and relations $\cup, \cap, \backslash, \subseteq, \supseteq, \subseteq^{*}$ and $\supseteq^{*}$ on elements of $\mathbb{P}(\kappa)$ will refer to the coordinatewise operations and relations; so, for example, $(f \backslash g)(\alpha)=f(\alpha) \backslash g(\alpha)$. The ordering on $\mathbb{P}(\kappa)$ is $\subseteq^{*}$ in the sense just defined; in other words, $f \subseteq^{*} f^{\prime}$ if and only if $f(\alpha) \subseteq^{*} f^{\prime}(\alpha)$ for each $\alpha \in \kappa$.

The relation $\subseteq^{*}$ on $\mathbb{P}(\kappa)$ is not actually an ordering but only a pre-ordering. However, it can easily be made into an ordering by identifying $f$ with $g$ if $f \subseteq^{*}$ $g \subseteq^{*} f$. If $\kappa$ is finite, then the resulting equivalence classes are countable, but otherwise they are uncountable. However, to avoid having to deal with equivalence classes, the pre-ordering on $\mathbb{P}(\kappa)$ will be used in the sequel.

Lemma 3.2. $\mathbb{P}(\kappa)$ is countably closed.

Proof. Given a sequence $\left\{f_{n}\right\}_{n \in \omega} \subseteq \mathbb{P}(\kappa)$ such that $f_{n} \subseteq^{*} f_{n+1}$ for each $n \in \omega$, choose inductively $k_{n}(\alpha)$ for $\alpha \in \kappa$ such that

$$
f_{\omega}(\alpha)=\bigcup_{n \in \omega} f_{n}(\alpha) \uparrow\left(\omega \backslash k_{n}(\alpha)\right)
$$

is a function for each $\alpha \in \kappa$. Now apply Lemma 3.1 to each coordinate.

Definition 3.3. For any $G$ that is a centred subset of $\mathbb{P}(\kappa)$, define $\Phi_{G}^{\alpha}: \mathcal{P}(\omega) /[\omega]^{<\aleph_{0}} \rightarrow \mathcal{P}(\omega) /[\omega]^{<\aleph_{0}}$ by

$$
\Phi_{G}^{\alpha}([X])= \begin{cases}{[\{g(\alpha)(i): i \in X\}]} & \text { if }(\exists g \in G)(X \subseteq \operatorname{domain}(g(\alpha))), \\ {[\omega \backslash\{g(\alpha)(i): i \in \omega \backslash X\}]} & \text { if }(\exists g \in G)(\omega \backslash X \subseteq \operatorname{domain}(g(\alpha))),\end{cases}
$$

and, in the case that $\kappa=1$, the superscript will be omitted. 
Genericity easily yields the following.

Lemma 3.3. If $G \subseteq \mathbb{P}(\kappa)$ is a generic filter on $\mathbb{P}(\kappa)$, then $\Phi_{G}^{\alpha}$ is a nontrivial automorphism of $\mathcal{P}(\omega) /[\omega]<\aleph_{0}$ and $\Phi_{G}^{\alpha} \neq \Phi_{G}^{\beta}$ unless $\alpha=\beta$.

An important fact is the result of Velickovic [9] that if $F$ is $\mathbb{P}$-generic over a model $V$ of PFA, then in $V[F]$, not only is there a nontrivial autohomeomorphism of $\beta \mathbb{N} \backslash \mathbb{N}$, but MA also holds. Indeed, no new subsets of the ground model of size $\aleph_{1}$ are added. It will be shown that a closer analysis of these models yields that there are few automorphisms of $\mathcal{P}(\omega) /[\omega]^{<\aleph_{0}}$ in them.

Notation 3.1. The notation $\mathbb{S}$ will be used to denote Silver forcing, which consists of partial functions from $\omega$ to 2 with co-infinite domain.

Definition 3.4. Given $\mathfrak{S} \subseteq \mathbb{S}$ define $\mathbb{S}(\mathfrak{S}) \subseteq \mathbb{S}$ to be the partial order consisting of all $s \in \mathbb{S}$ such that there is some $s^{\prime} \in \mathfrak{S}$ such that $s \subseteq^{*} s^{\prime}$. The ordering is defined to be $\subseteq$.

Definition 3.5. For $f$ and $g$ in $\mathbb{P}(\kappa)$ define $f \leq_{\kappa}^{*} g$ if and only if $f \subseteq^{*} g$ and $f(\alpha) \subseteq g(\alpha)$ for all but finitely many $\alpha \in \kappa$.

From Lemma 3.1 it follows that, given a sequence $\left\{f_{\xi}\right\}_{\xi \in \omega_{1}} \subseteq \mathbb{P}(\kappa)$, it will be useful to find an element $f \in \mathbb{P}$ such that $f_{\xi} \subseteq^{*} f$ for each $\xi \in \omega_{1}$. The following definition of a partial order is designed to do precisely this.

Definition 3.6. Given $\mathfrak{F} \subseteq \mathbb{P}(\kappa)$ define $\mathbb{P}(\mathfrak{F}) \subseteq \mathbb{P}(\kappa)$ to be the partial order consisting of all $f \in \mathbb{P}(\kappa)$ such that there is some $f^{\prime} \in \mathfrak{F}$ such that $f \leq_{\kappa}^{*} f^{\prime}$. The ordering on $\mathbb{P}(\mathfrak{F})$ is coordinatewise inclusion. Given $f \in \mathbb{P}(\kappa), A \subseteq \kappa$ and an integer $M$, let $f\langle A, M\rangle$ be the function defined by

$$
f\langle A, M\rangle(\alpha)= \begin{cases}f(\alpha)\left\lceil 2^{M}\right. & \text { if } \alpha \in A, \\ \emptyset & \text { if } \alpha \in \kappa \backslash A .\end{cases}
$$

Define $f[A, M]=f \backslash f\langle A, M\rangle$ and let $\mathbb{P}\langle A, M\rangle=\{f \in \mathbb{P}(\kappa): f\langle A, M\rangle=f\}$.

For the purposes of this paper, $\mathbb{P}(\mathfrak{F})$ and $\mathbb{S}(\mathfrak{S})$ can be thought of as amoeba forcings for $\mathbb{P}(\kappa)$ and $\mathbb{S}$ in the sense that the generic objects they produce yield elements of $\mathbb{P}(\kappa)$ and $\mathbb{S}$, respectively. The following two technical results contain the details of the fusion constructions on the amoeba partial orders that will be required in later arguments.

Lemma 3.4. Given $a \leq_{\kappa^{*}}^{*}$-directed family $\mathfrak{F} \in[\mathbb{P}(\kappa)]^{\aleph_{0}}$ and a countable partial order $\mathbb{Q}$, both belonging to a countable model $\mathfrak{M}$ of some large fragment of set theory, there is an $f \in \mathbb{P}(\kappa)$ such that

(1) $f^{\prime} \leq_{\kappa}^{*} f$ for all $f^{\prime} \in \mathfrak{F}$;

(2) for each $D \in \mathfrak{M}$ that is dense in $\mathbb{P}(\mathfrak{F}) \times \mathbb{Q}$, for each $q \in \mathbb{Q}$ and each finite set $A \subseteq \kappa \cap \mathfrak{M}$, there are arbitrarily large integers $M$ such that, given $h \in \mathbb{P}\langle A, M\rangle$, there is some $(p, \bar{q}) \in D$ such that $h \subseteq p, q \leq \bar{q}$ and $p[A, M] \subseteq f$.

Proof. The argument here is standard. Let $\left\{f_{n}\right\}_{n \in \omega}$ be a cofinal $\leq_{\kappa}^{*}$-increasing sequence in $\mathbb{P}(\mathfrak{F})$. Let $\mathfrak{D}$ be the collection of all dense subsets of $\mathbb{P}(\mathfrak{F}) \times \mathbb{Q}$ belonging to $\mathfrak{M}$, let $\left\{\left(D_{i}, q_{i}, A_{i}\right)\right\}_{i \in \omega}$ enumerate infinitely often $\mathfrak{D} \times \mathbb{Q} \times[\kappa \cap \mathfrak{M}]<\aleph_{0}$, and choose 
inductively a sequence of conditions $\left(p_{n}, \bar{q}_{n}\right) \in \mathfrak{M} \cap(\mathbb{P}(\mathfrak{F}) \times \mathbb{Q})$, finite sets $A_{n}^{*}$ and integers $M_{n}$ such that

$$
\begin{gathered}
A_{n}^{*} \cup A_{n+1} \subseteq A_{n+1}^{*}, \\
\left(\forall h \in \mathbb{P}\left\langle A_{n}^{*}, M_{n}\right\rangle\right)\left(h \cup p_{n}\left[A_{n}, M_{n}\right], \bar{q}_{n}\right) \in D_{n}, \\
\left(\forall \beta \in A_{n}\right)\left(\exists j<M_{n}\right) \mid\left[2^{j}, 2^{j+1}\right) \backslash \text { domain }\left(p_{n}(\beta)\right) \mid>n, \\
p_{n+1}\left\langle A_{n}^{*}, M_{n}\right\rangle=p_{n}\left\langle A_{n}^{*}, M_{n}\right\rangle, \\
p_{n} \subseteq p_{n+1}, \\
q_{n} \leq \bar{q}_{n}, \\
f_{n} \leq_{\kappa}^{*} p_{n} .
\end{gathered}
$$

Let $f=\bigcup_{n \in \omega} p_{n}$. It follows from Conditions (3.3), (3.4), (3.1) and (3.5) that $f \in \mathbb{P}(\kappa)$. From Condition (3.7), it follows that $f \supseteq p_{n} \geq_{\kappa}^{*} f_{n}$, and so Condition (1) is satisfied. To see that Condition (2) is satisfied use Conditions (3.2).

To see that the induction can be carried out, suppose that $p_{n}$ has been constructed. Let $A_{n+1}^{*}=A_{n+1} \cup A_{n}^{*}$. Choose $M_{n+1}$ so large that

$$
\left(\forall \beta \in A_{n+1}^{*}\right)\left(\exists j<M_{n+1}\right)\left|\left[2^{j}, 2^{j+1}\right) \backslash \operatorname{domain}\left(p_{n}(\beta)\right)\right|>n+1
$$

and let $\left\{h_{i}\right\}_{i=0}^{k}$ enumerate $\mathbb{P}\left\langle A_{n+1}^{*}, M_{n+1}\right\rangle$. Using the density of $D_{n+1}$ in $\mathbb{P}(\mathfrak{F}) \times \mathbb{Q}$, find a sequence of conditions $\left\{\left(p_{i}^{*}, q_{i}^{*}\right)\right\}_{i=0}^{k}$ such that

$$
\begin{gathered}
p_{i+1}^{*}\left[A_{n+1}^{*}, M_{n+1}\right] \supseteq p_{i}^{*}\left[A_{n+1}^{*}, M_{n+1}\right], \\
p_{i}^{*} \in D_{n+1}, \\
p_{i}^{*}\left\langle A_{n+1}^{*}, M_{n+1}\right\rangle=h_{i}, \\
q_{i}^{*} \leq q_{i+1}^{*}
\end{gathered}
$$

for each $i \leq k$. Let $\bar{q}_{n+1}=q_{k}^{*}$. Using the directedness of $\mathfrak{F}$ it is easy to find $p_{n+1} \supseteq$ $p_{k}^{*}$ such that Condition (3.7) is satisfied and, moreover, $p_{n+1}\left\langle A_{n+1}^{*}, M_{n+1}\right\rangle=$ $p_{n}\left\langle A_{n+1}^{*}, M_{n+1}\right\rangle$. This last requirement guarantees that Condition (3.3) is satisfied by the choice of $M_{n+1}$. The fact that $p_{k}^{*}\left[A_{n+1}^{*}, M_{n+1}\right] \subseteq p_{n+1}\left[A_{n+1}^{*}, M_{n+1}\right]$ guarantees that Conditions (3.2) and (3.5) are also satisfied.

Corollary 3.1. Given $a \leq_{\kappa}^{*}$-directed family $\mathfrak{F} \in[\mathbb{P}(\kappa)]^{\aleph_{0}}$ and a countable partial order $\mathbb{Q}$, both belonging to a countable model $\mathfrak{M}$ of some large fragment of set theory, there is an $f \in \mathbb{P}(\kappa)$ such that $f^{\prime} \leq_{\kappa}^{*} f$ for all $f^{\prime} \in \mathfrak{F}$ and, moreover, each $D \in \mathfrak{M}$ that is dense in $\mathbb{P}(\mathfrak{F}) \times \mathbb{Q}$ remains dense in $\mathbb{P}(\{\bar{f}\}) \times \mathbb{Q}$ for any $\bar{f} \in \mathbb{P}(\kappa)$ such that $f \leq_{\kappa}^{*} \bar{f}$.

Proof. Let $f$ be the element of $\mathbb{P}(\kappa)$ whose existence follows from Lemma 3.1, and suppose that $\bar{f} \in \mathbb{P}(\kappa)$ is such that $f \leq_{\kappa}^{*} \bar{f}$. If $D \in \mathfrak{M}$ is dense in $\mathbb{P}(\mathfrak{F}) \times \mathbb{Q}$ and $\left(f^{\prime}, q\right) \in \mathbb{P}(\{\bar{f}\}) \times \mathbb{Q}$, choose $A \in[\kappa]^{<\aleph_{0}}$ and $m$ so large that $f[A, m] \subseteq \bar{f}[A, m]$ and $f^{\prime}[A, m] \subseteq \bar{f}[A, m]$. Then, let $M \geq m$ be such that for each $h \in \mathbb{P}\langle A, M\rangle$ there is some $(p, \bar{q}) \in D$ such that $h \subseteq p, p \backslash h \subseteq f$ and $\bar{q} \geq q$. Then there is $(p, \bar{q}) \in D$ such that $f^{\prime}\langle A, M\rangle \subseteq p, p[A, M] \subseteq f[A, M] \subseteq \bar{f}[A, M]$ and $\bar{q} \geq q$. Since $f^{\prime}[A, M] \subseteq \bar{f}[A, M]$, it follows that $(p, \bar{q})$ is compatible with $\left(f^{\prime}, q\right)$.

Corollary 3.2. Given $\mathfrak{F} \in[\mathbb{P}(\kappa)]^{\aleph_{0}}$ and a countable partial order $\mathbb{Q}$, both belonging to a countable model $\mathfrak{M}$ of some large fragment of set theory, there is $f \in \mathbb{P}(\kappa)$ 
satisfying the conclusions of Lemma 3.4 and, moreover, for each $\mathbb{P}(\mathfrak{F})$-name, $\stackrel{\circ}{g} 1$ for an element of $\omega_{\omega}$ there is some $d \in \omega_{\omega}$ such that $1 \Vdash_{\mathbb{P}(\{f\})} " g \leq^{*} d "$. Moreover, if $f \leq_{\kappa}^{*} \bar{f}$, it is still true that $1 \Vdash_{\mathbb{P}(\{\bar{f}\})} " \stackrel{\circ}{g} \leq^{*} d "$.

Proof. The conclusion of Lemma 3.4 immediately yields a dominating function $d$ from $f$. To see this, let $D_{n}$ be the set of conditions deciding the value of $\stackrel{\circ}{g}(n)$, let $A_{n}$ and $M_{n}$ be such that, given $h \in \mathbb{P}\left\langle A_{n}, M_{n}\right\rangle$, there is some $p_{h} \in D_{n}$ such that $h \subseteq p_{h}, q \leq 1_{\mathbb{Q}}$ and $p_{h}[A, M] \subseteq f$, and let $d(n, h)$ be the value of $\stackrel{\circ}{g}(n)$ decided by $p_{h}$. Let

$$
d(n)=\max _{h \in \mathbb{P}\left\langle A_{n}, M_{n}\right\rangle} d(n, h)+1
$$

and observe that the role of $\mathbb{Q}$ is irrelevant. The final sentence follows from Corollary 3.1

Lemma 3.5. Given $\mathfrak{S} \in[\mathbb{S}]^{\aleph_{0}}$ and a countable partial order $\mathbb{Q}$, both belonging to a countable model $\mathfrak{M}$ of some large fragment of set theory, and $U \subseteq \omega$ such that $U \cap \operatorname{domain}(s)$ is finite for each $s \in \mathfrak{S}$, there is a comeagre set of partitions $U=U^{0} \cup U^{1}$ such that each $D \in \mathfrak{M}$ that is dense in $\mathbb{Q} \times \mathbb{S}(\mathfrak{S})$ remains predense in $\mathbb{Q} \times \mathbb{S}\left(\mathfrak{S} \cup\left\{0_{W \backslash Y} \cup 1_{Y}\right\}\right)$ for any choice of $a \in 2$ and $Y \subseteq W \subseteq U^{a}$.

Proof. This is the argument used to establish $\left({ }^{*} 1\right)$ on page 175 of $[3]$.

Notation 3.2. For the rest of this section, if $\Phi: \mathcal{P}(\omega) /[\omega]^{<\aleph_{0}} \rightarrow \mathcal{P}(\omega) /[\omega]<\aleph_{0}$ is an automorphism, then $\Phi^{*}$ will denote an arbitrarily chosen lifting of $\Phi$. In other words, $\Phi^{*}: \mathcal{P}(\omega) \rightarrow \mathcal{P}(\omega)$ is such that $\Phi^{*}(A) \in \Phi([A])$ for each $A \subseteq \omega$. This convention will be adhered to, even if $\Phi$ is only a forcing name for an automorphism of $\mathcal{P}(\omega) /[\omega]<\aleph_{0}$.

Lemma 3.6. Suppose that $\Phi$ is an automorphism of $\mathcal{P}(\omega) /[\omega]<\aleph_{0}$ in $V$. Let $\left\{A_{n}\right\}_{n \in \omega}$ and $A$ be subsets of the integers such that $A \supseteq^{*} A_{n}$ for each $n$ and there do not exist Borel functions $\left\{\psi_{n}: n \in \omega\right\}$ such that for all but a meagre set of $C \in \mathcal{P}(A) \cap V$ there is some $n \in \omega$ such that $\Phi^{*}(C) \triangle \psi_{n}(C) \subseteq \bigcup_{i \in k} \Phi^{*}\left(A_{i}\right)$ for some $k \in \omega$. If $V^{\prime}$ is obtained by adding a Cohen real to $V$, then, in $V^{\prime}$, there also do not exist Borel functions $\left\{\psi_{n}: n \in \omega\right\}$ such that for all but a meagre set of $C \in \mathcal{P}(A) \cap V$ there is some $n \in \omega$ such that $\Phi^{*}(C) \triangle \psi_{n}(C) \subseteq \bigcup_{i \in k} \Phi^{*}\left(A_{i}\right)$ for some $k \in \omega$.

Proof. Suppose that $V^{\prime}$ is obtained by forcing with the countable partial order $\mathbb{C}$ and that $\psi_{n}$ are $\mathbb{C}$-names for Borel functions such that for each $C \in \mathcal{P}(A) \cap V$ there is some $n \in \omega$ such that $\Phi^{*}(C) \triangle \psi_{n}(C) \subseteq \bigcup_{i \in k} \Phi^{*}\left(A_{i}\right)$ for some $k \in \omega$. For each integer $n$, let $G_{n}$ be a co-meagre set such that $\psi_{n}\left\lceil G_{n}\right.$ is continuous. Define

$$
\psi_{n}^{p}=\left\{(A, B): p \Vdash_{\mathbb{C}} \text { “ } \stackrel{\circ}{\psi}_{n}(A)=B \text { and } A \in \stackrel{\circ}{G}_{n} \text { " }\right\} .
$$

Let $D_{n}^{p}$ be the closure of the domain of $\psi_{n}^{p}$, and let $E_{n}^{p}$ be the closure of the interior of $D_{n}^{p}$-note that $D_{n}^{p} \backslash E_{n}^{p}$ is meagre since it is contained in the boundary of the closed set $D_{n}^{p}$. Let $f_{n}^{p}$ be the maximal extension of $\psi_{n}^{p}$ to a continuous function on a subset of $E_{n}^{p}$.

It must be the case that the domain of $f_{n}^{p}$ is co-meagre in $E_{n}^{p}$, because if the domain of $f_{n}^{p}$ is not co-meagre in $E_{n}^{p}$, then, because it is Borel, there must be some

\footnotetext{
${ }^{1}$ Observe that the claim is made only for those $\stackrel{\circ}{g} \in \mathfrak{M}$ that are $\mathbb{P}(\mathfrak{F})$ names and not for $\mathbb{P}(\mathfrak{F}) \times \mathbb{Q}$ names in general.
} 
open set $U \subset E_{n}^{p}$ such that the set of points in $U$ to which $\psi_{n}^{p}$ can be continuously extended is meagre in $U$. Since $p \Vdash " \psi_{n}^{p} \subset \psi_{n}$ " and because being a meagre Borel set is absolute, it must be that the set of points in $U$ to which $\psi_{n}$ can be continuously extended is also meagre in $U$. The reason is that the domain of $\psi_{n}^{p}$ is dense in $E_{n}^{p}$, and so it follows that the domain of $\psi_{n}^{p}$ is dense in $U$ and, moreover, not being a point to which a function can be continuously extended is an absolute property. This contradicts the fact that $G_{n}$ is co-meagre.

Now let

$$
M^{\prime}=\left(\bigcup_{n \in \omega \text { and } p \in \mathbb{C}} D_{n}^{p} \backslash E_{n}^{p}\right) \cup\left(\bigcup_{n \in \omega} E_{n}^{p} \backslash \operatorname{domain}\left(f_{n}^{p}\right)\right)
$$

and observe that $M^{\prime}$ is meagre. By the hypothesis on $V$ it follows that the set $S=$

$$
\left\{C \in \mathcal{P}(A):(\forall p \in \mathbb{C})(\forall k \in \omega) \psi_{n}^{p}(A) \text { is not defined or } \psi_{n}^{p}(A) \triangle \Phi^{*}(A) \not \subseteq \bigcup_{i \in k} \Phi^{*}\left(A_{i}\right)\right\}
$$

is a second category set. Hence, $S$ remains of second category after adding a Cohen real. Let $N$ be a meagre set such that $G_{n} \supseteq \mathcal{P}(\omega) \backslash N$ for each $n \in \omega$, let $M=M^{\prime} \cup N$ and choose $C \in S \backslash M$ in $V^{\prime}$. But then there are some $p \in \mathbb{C}$ and $k \in \omega$ such that $\psi_{n}^{p}(A) \triangle \Phi^{*}(A) \subseteq \bigcup_{i \in k} \Phi^{*}\left(A_{i}\right)$, which is a contradiction.

Theorem 3.1. If $V$ is a model of PFA and $F$ is $\mathbb{P}(\kappa)$ generic over $V$, then, in $V[F]$, given any automorphism $\Phi$ of $\mathcal{P}(\omega) /[\omega]^{<\aleph_{0}}$ and disjoint infinite sets $\left\{A_{n}\right\}_{n \in \omega}$,

$$
\begin{aligned}
& (\exists A)\left(\exists\left\{\psi_{n}\right\}_{n \in \omega}\right)(\forall n) A \supseteq^{*} A_{n} \text { and } \\
& \psi_{n}: \mathcal{P}(A) \rightarrow \mathcal{P}(\omega) \text { is a partial Borel function and } \\
& \quad(\forall X \in \mathcal{P}(A))(\exists m, k \in \omega) \Phi^{*}(X) \triangle \psi_{m}(X) \subseteq \bigcup_{i \leq k} \Phi^{*}\left(A_{i}\right) .
\end{aligned}
$$

Proof. Let $\Phi$ be an automorphism of $\mathcal{P}(\omega) /[\omega]^{<\aleph_{0}}$ in $V[F]$ and suppose that $\left\{A_{n}\right\}_{n \in \omega}$ are infinite pairwise disjoint sets. Without loss of generality, $\emptyset \Vdash_{\mathbb{P}(\kappa)}$ " $\stackrel{\circ}{\Phi}$ is an automorphism of $\mathcal{P}(\omega) /[\omega]<\aleph_{0}$ and 3.12 fails for $\left\{A_{n}\right\}_{n \in \omega}$ and $\Phi^{*}$ " and $\Phi^{*}\left(A_{i}\right) \cap \Phi^{*}\left(A_{j}\right)=\emptyset$ unless $i=j$. Let $G$ be a $V$-generic filter on a countably closed partial order, $\mathbb{D}$, which forces the existence of a $\diamond$-sequence $\left\{\mathfrak{M}_{\xi}\right\}_{\xi \in \omega_{1}}$.

As in [5], let $\mathfrak{F}=\left\{f_{\eta}\right\}_{\eta \in \omega_{1}}$ be a $\leq_{\kappa}^{*}$-increasing sequence in $V[G]$ such that

- for each $C \in V \cap \mathcal{P}(\omega)=V[G] \cap \mathcal{P}(\omega)$, the value of $\Phi^{*}(C)$ is decided by some $f_{\eta}$ with respect to forcing by $\mathbb{P}(\kappa)$;

- the partial order $\mathbb{P}(\mathfrak{F})$ is ccc;

- forcing with $\mathbb{P}(\mathfrak{F})$ preserves dominating families in $\omega_{\omega}$;

- for each $A \in V$ such that $A \supseteq^{*} A_{n}$ for each $n$, forcing with $\mathbb{P}(\mathfrak{F})$ preserves that there do not exist Borel functions $\left\{\psi_{n}: n \in \omega\right\}$ such that for all but a meagre set of $C \in \mathcal{P}(A) \cap V$ there are $n \in \omega$ and $k \in \omega$ such that $\Phi^{*}(C) \triangle \psi_{n}(C) \subseteq \bigcup_{i \in k} \Phi^{*}\left(A_{i}\right)$.

The first requirement is easily accomplished at successor steps by using $2^{\aleph_{0}}=\aleph_{1}$ and the fact that the forcing relation is not changed by modifying infinitely many coordinates of a condition by a finite amount. The second requirement is obtained by a standard $\diamond$ argument, while the third is a consequence of Corollary [3.2 The last requirement is accomplished by appealing to the Omitting Type Theorem, 
Lemma 2.1 on page 153 of [3] and applying it to the family of sentences $\sigma_{C}(x)$ parameterized by $C \in \mathcal{P}(A)$ which say that $x=\left(\left\{\psi_{n}: n \in \omega\right\}, M\right)$ where each $\psi_{n}: \mathcal{P}(A) \rightarrow \mathcal{P}(\omega)$ is a Borel function, $M$ is a meagre $F_{\sigma}$ subset of $\mathcal{P}(A)$, and either $C \in M$ or there is some $n \in \omega$ and $k \in \omega$ such that $\Phi^{*}(C) \triangle \psi_{n}(C) \subseteq \bigcup_{i \in k} \Phi^{*}\left(A_{i}\right)$. Lemma 3.6 asserts that Cohen forcing preserves that there is no $x$ such that $\sigma_{C}(x)$ holds for each $C \in \mathcal{P}(A)$. The Omitting Type Theorem allows the conclusion that there is an oracle $\mathfrak{N}$ such that forcing with any partial order that satisfies the $\mathfrak{N}-c c$ preserves that there is no $x$ such that $\sigma_{C}(x)$ holds for all $C \in \mathcal{P}(A)$; so it suffices to construct $\mathfrak{F}$ to satisfy the $\mathfrak{N}-c c$. So, it may be assumed that $\mathfrak{N}=\left\{\mathfrak{M}_{\eta}\right\}_{\eta \in \omega_{1}}$.

Let $\left\{d_{\xi}\right\}_{\xi \in \omega_{1}}$ be a $\leq^{*}$-increasing, dominating family in $\omega_{\omega}$ in $V[G]$. It may, without loss of generality, be assumed that the sets $A_{n}$ form a partition of $\omega$ and that $\bigcup_{n \in \omega} \Phi^{*}\left(A_{n}\right)=\omega$. Let $\Theta: \omega \rightarrow \omega \times \omega$ be a bijection in $V$ such that the range of $\Theta\left\lceil A_{n}\right.$ is precisely $\{(n, m)\}_{m \in \omega}$. For $\alpha \in \beta \in \omega_{1}$ define $W_{\alpha, \beta}=\Theta^{-1}\{(n, m)$ : $\left.d_{\alpha}(n) \leq m<d_{\beta}(n)\right\}$ and let $W_{\alpha, \omega_{1}}=\Theta^{-1}\left\{(n, m): d_{\alpha}(n) \leq m\right\}$. Observe that $W_{\alpha, \omega_{1}} \supseteq^{*} A_{n}$ for each $n \in \omega$ and $\alpha \in \omega_{1}$, while $W_{\alpha, \beta} \cap A_{n}$ is finite for each $n \in \omega$ and $\alpha \in \beta \in \omega_{1}$. Moreover, the sets $W_{\alpha, \beta}$ all belong to $V$.

Now, suppose that $H$ is a $V[G]$ generic subset of $\mathbb{P}(\mathfrak{F})$. In $V[G][H]$, let $\left\{\stackrel{\circ}{Y}_{\eta}\right\}_{\eta \in \omega_{1}}$ enumerate all possible names for reals with respect to hereditarily countable partial orders and let $\left\{\mathfrak{M}_{\eta}[H]\right\}_{\eta \in \omega_{1}}$ be an oracle given by $\diamond$ (which exists since $\mathbb{P}(\mathfrak{F})$ has the countable chain condition, is of size $\aleph_{1}$ and $\diamond$ holds in $V[G]$ ). It may also be assumed that $\left\{\stackrel{\circ}{Y}_{\xi}\right\}_{\xi \in \zeta} \subseteq \mathfrak{M}_{\zeta}[H]$ for each $\zeta \in \omega_{1}$, since this can be accomplished by simply enlarging the oracle's guesses. Construct in $V[G][H]$ a sequence of pairs of ordinals $\left\{\left(\alpha_{\eta}, \beta_{\eta}\right)\right\}_{\eta \in \omega_{1}}$ and pairs $\left\{\left(V_{\eta}, W_{\eta}\right)\right\}_{\eta \in \omega_{1}}$ such that $\alpha_{\eta} \in \beta_{\eta} \in \alpha_{\gamma}$ if $\eta \in \gamma$ and $V_{\eta} \subseteq W_{\eta} \subseteq W_{\alpha_{\eta}, \beta_{\eta}}$. Furthermore, it will be required that, letting $\mathbb{S}_{\zeta}=\mathbb{S}\left(\left\{0_{W_{\eta} \backslash V_{\eta}} \cup 1_{V_{\eta}}\right\}_{\eta \in \zeta}\right)$

$$
1 \Vdash_{\mathbb{S}_{\zeta}} \text { " } \stackrel{\circ}{\Phi}^{*}\left(V_{\eta}\right) \triangle\left(\stackrel{\circ}{Y}_{\mu} \cap \stackrel{\circ}{\Phi^{*}}\left(W_{\eta}\right)\right) \not \subseteq \bigcup_{i \in k} \stackrel{\circ}{\Phi^{*}}\left(A_{i}\right) "
$$

for each $\mu \leq \eta \in \zeta$ and any $k \in \omega$. In particular,

$$
1 \Vdash_{\mathbb{S}_{\zeta}} \text { “ } \stackrel{\circ}{\Phi}^{*}\left(V_{\eta}\right) \triangle\left(\stackrel{\circ}{Y_{\mu}} \cap \stackrel{\circ}{\Phi^{*}}\left(W_{\eta}\right)\right) \not \equiv * \emptyset "
$$

and so, if this induction can be carried out, then, using PFA, it is possible to argue just as in [5]. The main point is that the set $H$ is generic for $\mathbb{P}(\mathfrak{F})$, which is the amoeba forcing of $\mathbb{P}(\kappa)$, and produces a condition $f^{*}$ in $\mathbb{P}(\kappa)$ using Lemma 3.1 Moreover, $f^{*}$ decides all that it is required to know about $\Phi^{*}$. In other words, the argument $\left[2\right.$ from [5] uses PFA to produce a subset of $\mathcal{P}(\omega) /[\omega]<\aleph_{0}$ of size $\aleph_{1}$ which witnesses that $\Phi$ cannot be extended to a certain element of $\mathcal{P}(\omega) /[\omega]<\aleph_{0}$ and $f^{*}$ forces all the necessary values of $\Phi^{*}$ on this set of size $\aleph_{1}$. Hence $f^{*}$ contradicts that $\Phi$ is an automorphism of $\mathcal{P}(\omega) /[\omega]<\aleph_{0}$ in spite of the fact that $\emptyset$ forces this.

Now, suppose that the induction has been completed for all ordinals less than $\eta$. Let $\alpha_{\eta} \in \omega_{1}$ be so large that $\beta_{\zeta} \in \alpha_{\eta}$ for each $\zeta \in \eta$. Use Lemma 3.5 with $\mathbb{Q}$ being a trivial partial order, to find a co-meagre set $\mathfrak{E} \subseteq \mathcal{P}\left(W_{\alpha_{\eta}, \omega_{1}}\right)$ such that if $W \in \mathfrak{E}$, then all predense sets of $\mathbb{S}_{\eta}$ belonging to $\mathfrak{M}_{\eta}[H]$ remain predense in

$$
\mathbb{S}\left(\left\{0_{W_{\zeta} \backslash V_{\zeta}} \cup 1_{V_{\zeta}}\right\}_{\zeta \in \eta} \cup\left\{0_{W_{\eta} \backslash V_{\eta}} \cup 1_{V_{\eta}}\right\}\right)
$$

\footnotetext{
${ }^{2}$ The argument is reproduced in more detail, but in a slightly different context, in the proof of Theorem 4.1
} 
regardless of the choice of $W_{\eta}$ or $V_{\eta}$ provided that $V_{\eta} \subseteq W_{\eta} \subseteq W$ or $V_{\eta} \subseteq W_{\eta} \subseteq$ $W_{\alpha_{\eta}, \omega_{1}} \backslash W$. Since dominating families in $V$ remain dominating in $V[G][H]$, it follows that the reals of $V$ remain of second category in $V[G][H]$; hence, it is possible to choose $W \subseteq W_{\alpha_{\eta}, \omega_{1}}$ belonging to $V \cap \mathfrak{E}$.

Let $W^{0}=W$ and $W^{1}=W_{\alpha_{\eta}, \omega_{1}} \backslash W$. For $i \in 2, p: D \rightarrow 2$ such that $p \in \mathbb{S}_{\eta}$, $k \in \omega$ and $\xi \in \eta$, define a partial function

$$
\psi_{i, p, k, \xi}: \mathcal{P}\left(W^{i}\right) \rightarrow \mathcal{P}\left(\bigcup_{j=k}^{\infty} \Phi^{*}\left(A_{j}\right) \cap \Phi^{*}\left(W^{i}\right)\right)
$$

by $\psi_{i, p, k, \xi}(C)=C^{\prime}$ if and only if

$$
\begin{aligned}
& \left(\forall n \in C^{\prime}\right)\left(\forall p^{\prime} \in \mathbb{S}_{\eta}\right) \text { if } p \cup p^{\prime} \cup 0_{W^{i} \backslash(C \cup D)} \cup 1_{C \backslash D} \text { is a function, then } \\
& \left(\exists p^{\prime \prime} \supseteq p \cup p^{\prime}\right) p^{\prime \prime} \Vdash_{\mathbb{S}_{\eta}} \text { " } n \in \stackrel{\circ}{Y}_{\xi} \text { " and } p^{\prime \prime} \cup 0_{W^{i} \backslash(C \cup D)} \cup 1_{C \backslash D} \text { is a function }
\end{aligned}
$$

and

$$
\begin{gathered}
\left(\forall n \in\left(\bigcup_{j=k}^{\infty} \Phi^{*}\left(A_{j}\right) \cap \Phi^{*}\left(W^{i}\right)\right) \backslash C^{\prime}\right)\left(\forall p^{\prime} \in \mathbb{S}_{\eta}\right) \\
\quad \text { if } p \cup p^{\prime} \cup 0_{W^{i} \backslash(C \cup D)} \cup 1_{C \backslash D} \text { is a function, then } \\
\left(\exists p^{\prime \prime} \supseteq p \cup p^{\prime}\right) p^{\prime \prime} \Vdash_{\mathbb{S}_{\eta}} \text { " } n \notin \stackrel{\circ}{Y} \text { ” " and } p^{\prime \prime} \cup 0_{W^{i} \backslash(C \cup D)} \cup 1_{C \backslash D} \text { is a function. }
\end{gathered}
$$

Define $\psi_{p, q, k, \xi, \zeta}: \mathcal{P}\left(W_{\alpha_{\eta}}\right) \rightarrow \mathcal{P}\left(\bigcup_{j=k}^{\infty} \Phi^{*}\left(A_{j}\right)\right)$ by

$$
\psi_{p, q, k, \xi, \zeta}(C)=\psi_{0, p, k, \xi}\left(C \cap W^{0}\right) \cup \psi_{1, q, k, \zeta}\left(C \cap W^{1}\right),
$$

and observe that the functions $\psi_{p, q, k, \xi, \zeta}$ are all Borel. Hence there is some $C \in V$ such that

$$
\psi_{p, q, k, \xi, \zeta}(C) \triangle \Phi^{*}(C) \nsubseteq \bigcup_{j \in k} \Phi^{*}\left(A_{j}\right)
$$

for all $p, q, k, \xi$ and $\zeta$. It follows that there is some $i \in 2$ such that

$$
\psi_{i, p, k, \xi}\left(C \cap W^{i}\right) \triangle \Phi^{*}\left(C \cap W^{i}\right) \not \subseteq \bigcup_{j \in k} \Phi^{*}\left(A_{j}\right) \cap \Phi^{*}\left(W^{i}\right)
$$

for all $p, k$ and $\xi$. Fix $i \in 2$ to be such that (3.16) is satisfied for all $p, k$ and $\xi$.

Let $\left\{\left(p_{j}, \xi_{j}\right)\right\}_{j \in \omega}$ enumerate $\mathbb{S}_{\eta} \times \eta$ infinitely often and let $D_{j}$ be the domain of $p_{j}$. For each $j$ there is some $m_{j} \in \bigcup_{n=j}^{\infty} \Phi^{*}\left(A_{n}\right) \cap \Phi^{*}\left(W^{i}\right)$ such that if $\psi_{i, p_{j}, j, \xi_{j}}\left(C \cap W^{i}\right)$ is defined, then $m_{j} \in \psi_{i, p_{j}, j, \xi_{j}}\left(C \cap W^{i}\right) \triangle \Phi^{*}\left(C \cap W^{i}\right)$. In the case that $\psi_{i, p_{j}, j, \xi_{j}}\left(C \cap W^{i}\right)$ is defined, by appealing to (3.14) or (3.15), it is possible to find $p \in \mathbb{S}_{\eta}$ such that

$$
\begin{gathered}
p \supseteq p_{j}, \\
p \Vdash_{\mathbb{S}_{\eta}} \text { “ } m_{j} \in \stackrel{\circ}{Y_{\xi} \triangle} \stackrel{\circ}{\Phi^{*}}\left(C \cap W^{i}\right) \backslash \bigcup_{n \in j} \stackrel{\circ}{\Phi^{*}}\left(A_{n}\right) ", \\
p \cup 0_{W^{i} \backslash\left(C \cup D_{j}\right)} \cup 1_{C \backslash D_{j}} \text { is a function. }
\end{gathered}
$$

Furthermore, observe that if $\psi_{i, p_{j}, j, \xi_{j}}\left(C \cap W^{i}\right)$ is not defined, then there is some $m_{j} \in \bigcup_{k=j}^{\infty} \Phi^{*}\left(A_{k}\right) \cap \Phi^{*}\left(W^{i}\right)$ such that neither (3.14) nor (3.15) holds. To be precise,

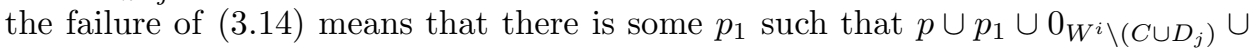
$1_{C \backslash D_{j}}$ is a function, but there is no $p^{\prime \prime} \supseteq p \cup p_{1}$ such that $p^{\prime \prime} \Vdash_{\mathbb{S}_{\eta}}$ " $n \in \stackrel{\circ}{Y_{\xi}}$ " and 
$p^{\prime \prime} \cup 0_{W^{i} \backslash\left(C \cup D_{j}\right)} \cup 1_{C \backslash D_{j}}$ is a function. Similarly, the failure of (3.15) means that there is some $p_{2}$ such that $p \cup p_{2} \cup 0_{W^{i} \backslash\left(C \cup D_{j}\right)} \cup 1_{C \backslash D_{j}}$ is a function, but there is no $p^{\prime \prime} \supseteq p \cup p_{2}$ such that $p^{\prime \prime} \Vdash_{\mathbb{S}_{\eta}}$ " $n \notin \stackrel{\circ}{Y_{\xi}}$ " and $p^{\prime \prime} \cup 0_{W^{i} \backslash\left(C \cup D_{j}\right)} \cup 1_{C \backslash D_{j}}$ is a function. However, the fact that $\stackrel{\circ}{Y}_{\xi} \in \mathfrak{M}_{\eta}[H]$ implies that the sets of conditions deciding membership in $\stackrel{\circ}{Y}_{\xi}$ are dense in $\mathbb{S}_{\eta}$ and hence in

$$
\mathbb{S}\left(\left\{0_{W_{\zeta} \backslash V_{\zeta}} \cup 1_{V_{\zeta}}\right\}_{\zeta \in \eta} \cup\left\{0_{W_{\eta} \backslash V_{\eta}} \cup 1_{V_{\eta}}\right\}\right)
$$

as well. So there must be $\bar{p}_{1}$ and $\bar{p}_{2}$ such that

- $\bar{p}_{1} \supseteq p \cup p_{1}$ and $\bar{p}_{2} \supseteq p \cup p_{2}$;

- $\bar{p}_{1} \Vdash_{\mathbb{S}_{\eta}}$ " $n \notin \stackrel{\circ}{Y}$ " " and $\bar{p}_{2} \Vdash_{\mathbb{S}_{\eta}}$ " $n \in \stackrel{\circ}{Y_{\xi}}$ ";

- $\bar{p}_{1} \cup 0_{W^{i} \backslash\left(C \cup D_{j}\right)} \cup 1_{C \backslash D_{j}}$ and $\bar{p}_{2} \cup 0_{W^{i} \backslash\left(C \cup D_{j}\right)} \cup 1_{C \backslash D_{j}}$ are both functions.

This makes it easy to once again find $p \in \mathbb{S}_{\eta}$ satisfying conditions (3.17), (3.18) and (3.19).

Since no unbounded reals are added by $H$ it is possible to find $Z \in V$ such that $Z \supseteq\left\{m_{j}\right\}_{j \in \omega}$ and such that $Z \cap \Phi^{*}\left(A_{n}\right)$ is finite for each $n$. It follows that there is some $\beta_{\eta}$ such that $\Phi^{*}\left(W_{\alpha_{\eta}, \beta_{\eta}}\right) \supseteq Z$. Let $W_{\eta}=W^{i} \cap W_{\alpha_{\eta}, \beta_{\eta}}$ and $V_{\eta}=C$.

To see that the pair $\left(V_{\eta}, W_{\eta}\right)$ satisfies the required conditions, let $q \in \mathbb{S}_{\eta+1}$, $k \in \omega$ and $\xi \in \eta+1$. It is possible to find $p: D \rightarrow 2$ such that $p \in \mathbb{S}_{\eta}$ and $q=p \cup 0_{W_{\eta} \backslash\left(V_{\eta} \cup D\right)} \cup 1_{V_{\eta} \backslash D}$. Let $j>k$ be such that $(p, \xi)=\left(p_{j}, \xi_{j}\right)$. By construction, there is some $p^{\prime} \in \mathbb{S}_{\eta}$ such that conditions (3.17), (3.18) and (3.19) hold for the case $j$. In particular, by noting that $D=D_{j}, p^{\prime} \cup p \cup 0_{W_{\eta} \backslash\left(V_{\eta} \cup D\right)} \cup 1_{V_{\eta} \backslash D} \supseteq p^{\prime} \cup q$ is a function belonging to $\mathbb{S}_{\eta+1}$ and $p^{\prime} \Vdash_{\mathbb{S}_{\eta}}$ " $m_{j} \in \stackrel{\circ}{Y}_{\xi} \triangle \stackrel{\circ}{\Phi}^{*}\left(C \cap W^{i}\right) \backslash \bigcup_{n \in j} \stackrel{\circ}{*}^{*}\left(A_{n}\right)$ ". Hence, (3.13) holds, as required.

Corollary 3.3. In the model $V[F]$, the ideal $\mathcal{T}(\Phi)$ is a dense $P$-ideal for every automorphism $\Phi$ of $\mathcal{P}(\omega) /[\omega]<\aleph_{0}$.

Proof. Let $\left\{A_{i}\right\}_{i \in \omega}$ be disjoint sets in the ideal $\mathcal{T}(\Phi)$ and let this fact be witnessed by $g_{i}: A_{i} \rightarrow \omega$. From Theorem 3.1 it follows that there is $A$ such that $A \supseteq^{*} A_{n}$ for each $n$ and there are Borel functions $\psi_{n}$ such that for all but a meagre set of $X \in \mathcal{P}(A)$ there are $m$ and $k$ such that $\Phi^{*}(X) \triangle \psi_{m}(X) \subseteq \Phi^{*}\left(\bigcup_{i \in k} A_{i}\right)$. Define

$$
\psi_{i, j}(X)=\left(\bigcup_{m \in i} g_{m}\left(X \cap A_{m}\right)\right) \cup\left(\psi_{j}(X) \backslash \Phi^{*}\left(\bigcup_{m \in i} A_{m}\right)\right) .
$$

Lemma 2.1 applied to the Borel functions $\left\{\psi_{i, j}\right\}_{i, j \in \omega}$ yields that $A \in \mathcal{T}(\Phi)$.

To see that $\mathcal{T}(\Phi)$ is dense is immediate: given any set $X \subseteq \omega$, let $\left\{A_{n}\right\}_{n \in \omega}$ be disjoint infinite subsets of $X$ and apply Theorem 3.1 to find $A$ such that $A \supseteq^{*} A_{n}$ for each $n$ and there are Borel functions $\psi_{n}$ such that for all $Y \in \mathcal{P}(A)$ there are $m$ and $k$ such that

$$
\Phi^{*}(Y) \triangle \psi_{m}(X) \subseteq \Phi^{*}\left(\bigcup_{i \in k} A_{i}\right) .
$$

Now choose an infinite $A^{\prime} \subseteq A$ such that $A \cap A_{n}$ is finite for all $n$. It follows that $\Phi^{*}(Y) \triangle \psi_{m}(X)$ is finite for each $Y \subseteq A^{\prime}$ and hence, from Lemma [2.1, it can be concluded that $\Phi$ is trivial on $A^{\prime}$. 


\section{WHY THERE ARE FEW AUTOMORPHISMS}

The next step is to establish the main result, which is that there are no more than $\kappa^{\aleph_{0}}$ automorphisms of $\mathcal{P}(\omega) /[\omega]^{<\aleph_{0}}$ in the model obtained by forcing with $\mathbb{P}(\kappa)$ over a model of PFA. In order to prove this, one is tempted to proceed by asserting that every automorphism is definable from the generic set. To establish this, suppose that $\emptyset$ forces, with respect to $\mathbb{P}(\kappa)$, that for some automorphism $\Phi$ there is no Borel definition of $\Phi$ using the generic set as a parameter. Add a $\diamond$ sequence to the initial model of PFA, and note that, since no new reals have been added, it is still forced, with respect to $\mathbb{P}(\kappa)$, that no Borel definition exists. The first difficulty is that it is no longer possible to iterate the two partial orders $\mathbb{P}(\mathfrak{F})$ and $\mathbb{S}(\mathfrak{S})$, since the first does add reals and so may add a Borel definition. Hence, the iteration must be replaced by a product. The induction involves simultaneously adding increasing conditions $f_{\eta}$ and almost disjoint sets $W_{\eta}$ such that $f_{\eta} \Vdash_{\mathbb{P}(\kappa)}$ " $W_{\eta} \in \mathcal{T}(\Phi)$ ". If the construction can be carried out, then, as usual, $\Phi$ cannot be extended. However, if the induction stops at stage $\zeta$, then this means that for each set $W \in \mathcal{T}(\Phi)$ that is almost disjoint from each $W_{\xi}$ for $\xi \in \zeta$, and for every $V \subseteq W$ there is some $\xi \in \zeta$ such that $\stackrel{\circ}{Y}_{\xi} \cap g(W)$ is forced to be almost equal to $g(V)$ where $g: W \rightarrow \omega$ is a one-to-one function witnessing that $W \in \mathcal{T}(\Phi)$. Now the name $\stackrel{\circ}{Y}_{\xi}$ can be used to determine $g$. Using that $\mathcal{T}(\Phi)$ is a $P$-ideal it is possible to find a single $\xi \in \zeta$ that works for a dense set of $W$. Were it not for the fact that $g(W)$ needs to be known in order to use $\stackrel{\circ}{Y}_{\xi}$ to determine $g$, this would finish the argument. This difficulty needs to be dealt with by resorting to arguments from [6].

In the following theorem, the term "Borel" is used in the context of subsets of $\mathbb{P}(\kappa) \times \mathcal{P}(\omega)$; so its meaning should be explained. First, note that $\mathbb{P}$ is a $G_{\delta}$ subset of $\omega_{\omega}$; so it inherits a Polish topology in a natural way. This yields a natural Polish topology on $\mathbb{P}^{A} \times \mathcal{P}(\omega)$ for any countable set $A$. A function $B$ from $\mathbb{P}(\kappa) \times \mathcal{P}(\omega)$ to some Polish space will be said to be Borel if there is some countable set $A \subseteq \kappa$ and a Borel function $B^{*}$ defined on $\mathbb{P}^{A} \times \mathcal{P}(\omega)$ such that $B(f)=B^{*}(f \uparrow A)$ for all $f \in \mathbb{P}(\kappa)$.

Theorem 4.1. If $V$ is a model of PFA and $F$ is $\mathbb{P}(\kappa)$ generic over $V$ and $\Phi \in V[F]$ is an automorphism of $\mathcal{P}(\omega) /[\omega]^{<\aleph_{0}}$, then there is a Borel function $B$ belonging to $V$ and mapping $\mathbb{P}(\kappa) \times \mathcal{P}(\omega)$ to partial bijections of $\omega$ such that for all $W \in[\omega]^{\aleph_{0}}$ and $f \in F$ there is $\bar{W} \in[W]^{\aleph_{0}}$ and $\bar{f} \in F$ such that $\bar{f} \supseteq f$ and

$$
(\forall Z \subseteq \bar{W}) \Phi(Z) \equiv{ }^{*} B(\bar{f}, \bar{W})(Z) .
$$

Moreover $B(f, X) \subseteq B\left(f^{\prime}, X^{\prime}\right)$ provided that $B(f, X)$ and $B\left(f^{\prime}, X^{\prime}\right)$ are nonempty and $X \subseteq X^{\prime}$ and $f \supseteq f^{\prime}$.

Proof. Suppose that $\emptyset$ forces, with respect to $\mathbb{P}(\kappa)$, that for some automorphism $\Phi$ there is no $B$ satisfying the requirements of the theorem. As in the proof of Theorem 3.1. let $G \subseteq \mathbb{D}$ and add a $\diamond$ sequence $\left\{\mathfrak{M}_{\xi}\right\}_{\xi \in \omega_{1}}$ to $V$, the initial model of PFA, and note that, since no new reals have been added, it is still forced, with respect to $\mathbb{P}(\kappa)$, that no $B$ exists. For notational purposes only, fix an enumeration $\left\{\stackrel{\circ}{Y_{\xi}}\right\}_{\xi \in \omega_{1}}$ of all possible countable names (with respect to any partial order whose

\footnotetext{
${ }^{3}$ Note that in the conclusion of Theorem 4.1, it is not claimed that $B(f, X)$ is invariant under finite changes to $f$.
} 
elements are hereditarily countable) for subsets of $\omega$. It may also be assumed that $\left\{\stackrel{\circ}{Y}_{\xi}\right\}_{\xi \in \zeta} \subseteq \mathfrak{M}_{\zeta}$ for each $\zeta \in \omega_{1}$, since this can be accomplished by simply enlarging $\mathfrak{M}_{\zeta}$.

In $V[G]$ construct, if possible, a sequence $\left\{\left(f_{\xi}, W_{\xi}, V_{\xi}\right)\right\}_{\xi \in \omega_{1}}$ such that, letting $s_{\xi}=0_{W_{\xi} \backslash V_{\xi}} \cup 1_{V_{\xi}}$ and $\mathbb{P}_{\eta}=\mathbb{P}\left(\left\{f_{\xi}\right\}_{\xi \in \eta}\right) \times \mathbb{S}\left(\left\{s_{\xi}\right\}_{\xi \in \eta}\right)$, the following conditions hold:

(1) For each $\eta, f_{\eta} \Vdash_{\mathbb{P}(\kappa)}$ " $W_{\eta} \in \mathcal{T}(\stackrel{\circ}{\Phi})$ ".

(2) The partial functions $\left\{f_{\xi}\right\}_{\xi \in \omega_{1}}$ are increasing with respect to $\leq_{\kappa}^{*}$, while $\left\{W_{\xi}\right\}_{\xi \in \omega_{1}}$ form an almost-disjoint family.

(3) For each $\eta \in \omega_{1}$ there are $A_{\eta} \subseteq \omega$ and $B_{\eta} \subseteq \omega$ such that

$$
f_{\eta} \Vdash_{\mathbb{P}(\kappa)} \text { " } \stackrel{\circ}{\Phi^{*}}\left(W_{\eta}\right)=A_{\eta} \text { and } \stackrel{\circ}{\Phi^{*}}\left(V_{\eta}\right)=B_{\eta} "
$$

and for each $\zeta \in \eta$,

$$
(\emptyset, \emptyset) \Vdash_{\mathbb{P}_{\eta}} " A_{\eta} \cap \stackrel{\circ}{Y_{\zeta}} \not \equiv * B_{\eta} " .
$$

(4) If $D \in \mathfrak{M}_{\eta}$ is a dense subset of $\mathbb{P}_{\eta}$, then $D$ is a dense subset of $\mathbb{P}_{\zeta}$ for each $\zeta \geq \eta$.

Observe that since $\stackrel{\circ}{Y}_{\zeta} \in \mathfrak{M}_{\eta}$ if $\zeta \in \eta$, it follows that the dense sets required to decide membership in $\stackrel{\circ}{Y}_{\zeta}$ all belong to $\mathfrak{M}_{\eta}$. Since $A_{\eta}$ and $B_{\eta}$ belong to $V$, it follows that the dense subsets of $\mathbb{P}_{\eta}$ required to guarantee condition (4.2) are subsets of $\mathfrak{M}_{\eta}$, but not necessarily elements of $\mathfrak{M}_{\eta}$. Nevertheless, since condition (4.2) only depends, in an absolute way, on the structure of the dense sets deciding membership in $\stackrel{\circ}{Y_{\zeta}}$, it follows from the last induction hypothesis that if $\mathbb{P}_{\eta}$ satisfies condition (4.2), then so does $\mathbb{P}_{\zeta}$ for each $\zeta \geq \eta$.

If this construction succeeds, then it is possible to proceed as in 4 to prove that forcing with $\mathbb{P}_{\omega_{1}}$ adds a set to which the partial automorphism $\Phi$ cannot be extended. In particular, if $H_{1} \times H_{2}$ is $\mathbb{P}_{\omega_{1}}$ generic, then, setting $X=\bigcup_{h \in H_{2}} h^{-1}\{1\}$, it follows that $X \cap W_{\xi} \equiv{ }^{*} V_{\xi}$ for each $\xi \in \omega_{1}$, but in $V[G]\left[H_{1} \times H_{2}\right]$ for every $Y \subseteq \omega$ there is $\eta \in \omega_{1}$ such that for each $\beta>\eta$,

$$
f_{\beta} \Vdash_{\mathbb{P}(\kappa)} \text { " } \stackrel{\circ}{\Phi^{*}}\left(W_{\beta}\right)=A_{\beta} \text { and } \stackrel{\circ}{\Phi^{*}}\left(V_{\beta}\right)=B_{\beta} "
$$

and $(\emptyset, \emptyset) \Vdash_{\mathbb{P}_{\beta}}$ " $A_{\beta} \cap \stackrel{\circ}{Y} \not{ }^{*} B_{\beta}$ " for each $\beta \geq \eta$. Just as in [4], it is possible to define a relation $R$ on $\omega_{1}$ by letting $R(\xi, \eta)$ hold if and only if

$$
\left(B_{\eta} \cap\left(A_{\xi} \backslash B_{\xi}\right)\right) \cup\left(B_{\xi} \cap\left(A_{\eta} \backslash B_{\eta}\right)\right) \neq \emptyset .
$$

It is easy to see that this is a semi-open relation-as defined by Abraham, Rubin and Shelah in [1. (or open as defined by Todorcevic in 7]) - and that, moreover, there is no $S \in\left[\omega_{1}\right]^{\aleph_{1}}$ such that $[S]^{2} \cap R=\emptyset$. Hence, by the results of [1], there is a proper partial order $\mathbb{K}$ that adds a set $S \in\left[\omega_{1}\right]^{\aleph_{1}}$ such that $[S]^{2} \subseteq R$. This makes the fact that $\Phi \uparrow V[G]\left[H_{1} \times H_{2}\right]$ cannot be extended to the set $X$ absolute. The reason for this is that if there were a set $Y$ such that $\Phi \uparrow V[G]\left[H_{1} \times H_{2}\right]([X])$ could be defined to be $[Y]$, then it would be the case that $Y \cap \Phi^{*}\left(W_{\xi}\right) \equiv^{*} \Phi^{*}\left(V_{\xi}\right)$ for each $\xi \in S$. But then there would be an uncountable set $S^{\prime} \subseteq S$, as well as $J \in \omega$, such that $Y \cap \Phi^{*}\left(W_{\xi}\right) \backslash J=\Phi^{*}\left(V_{\xi}\right) \backslash J$ for each $\xi \in S^{\prime}$. It would follow that $\Phi^{*}\left(V_{\xi}\right) \backslash J \subseteq Y$ and that $\left(\Phi^{*}\left(W_{\xi}\right) \backslash \Phi^{*}\left(V_{\xi}\right)\right) \backslash J \subseteq \omega \backslash Y$ for each $\xi \in S^{\prime}$. Choosing $\xi$ and $\zeta$ in $S^{\prime}$ such that $\Phi^{*}\left(V_{\xi}\right) \cap J=\Phi^{*}\left(V_{\zeta}\right) \cap \bar{J}$ and $\Phi^{*}\left(W_{\xi}\right) \cap J=\Phi^{*}\left(W_{\zeta}\right) \cap J$ would then yield the desired contradiction. 
The iteration $\mathbb{D} * \mathbb{P}_{\omega_{1}} * \mathbb{K}$ is proper, and only $\aleph_{1}$ dense sets in it need be met in order to obtain $S$ and the set $X$ such that $\Phi$ cannot be extended to include $[X]$ in its domain. Using PFA, choose a filter $\Gamma \subseteq \mathbb{D} * \mathbb{P}_{\omega_{1}} * \mathbb{K}$ that meets all of the relevant dense sets and let $f_{\Gamma}$ be the element of $\mathbb{P}(\kappa)$ obtained by intersecting $\Gamma$ with $\mathbb{P}\left(\left\{f_{\xi}\right\}_{\xi \in \omega_{1}}\right)$ - the first coordinate of $\mathbb{P}_{\omega_{1}}$-and then appealing to Lemma 3.1 Let $X_{\Gamma}$ be obtained by intersecting $\Gamma$ with $\mathbb{S}\left(\left\{s_{\xi}\right\}_{\xi \in \omega_{1}}\right)$, the second coordinate of $\mathbb{P}_{\omega_{1}}$. The intersection of $\Gamma$ with $\mathbb{K}$ yields a set which witnesses that the fragment of $\Phi$ determined by $f_{\Gamma}$ cannot be extended to include $\left[X_{\Gamma}\right]$ in its domain. It follows that $f_{\Gamma} \Vdash_{\mathbb{P}(\kappa)}$ " $\stackrel{\circ}{\Phi}$ does not extend to $\left[X_{\Gamma}\right]$ ". This contradicts that $\emptyset \Vdash_{\mathbb{P}(\kappa)}$ " $\stackrel{\circ}{\Phi}$ is an automorphism of $\mathcal{P}(\omega) /[\omega]<\aleph_{0} "$.

Hence, suppose that the induction stops at stage $\eta$ and that $\mathfrak{M}=\mathfrak{M}_{\eta}$ is the countable model presented by the $\diamond$ sequence at that stage. Using Corollary [3.1] applied to $\mathfrak{M}$, it is possible to find $f^{*} \in \mathbb{P}(\kappa)$ such that $f_{\zeta} \leq_{\kappa}^{*} f^{*}$ for each $\zeta \in \eta$ and for any $f^{\prime}$ such that $f^{*} \leq^{*} f^{\prime}$ where every $D \in \mathfrak{M}$ that is dense in $\mathbb{P}_{\eta}$ remains dense in $\mathbb{P}\left(\left\{f^{\prime}\right\}\right) \times \mathbb{S}\left(\left\{s_{\zeta}\right\}_{\zeta \in \eta}\right)$. Now apply Corollary 3.3] and the fact that $\mathbb{P}(\kappa)$ adds no new reals to find $\bar{f}_{\eta} \supseteq f^{*}, \bar{W}$ and $\varphi$ such that $\bar{W} \supseteq{ }^{*} W_{\zeta}$ for each $\zeta \in \eta$ and $\bar{f}_{\eta} \Vdash_{\mathbb{P}(\kappa)} " \varphi: \bar{W} \rightarrow \omega$ induces $\stackrel{\circ}{\Phi}$ ". Recall that the requirement $\bar{f}_{\eta} \supseteq f^{*}$ poses no problem because forcing with $\mathbb{P}(\kappa)$ is stable under finite modification of finitely many coordinates. Next, use Lemma 3.5 to find a partition $\omega \backslash \bar{W}=U^{0} \cup U^{1}$ such that for any $a \in 2$ and any $W \subseteq U^{a}$ and any $V \subseteq W$, every $D \in \mathfrak{M}$ that is dense in $\mathbb{P}\left(\left\{\bar{f}_{\eta}\right\}\right) \times \mathbb{S}\left(\left\{s_{\zeta}\right\}_{\zeta \in \eta}\right)$ remains dense in $\mathbb{P}\left(\left\{\bar{f}_{\eta}\right\}\right) \times \mathbb{S}\left(\left\{s_{\zeta}\right\}_{\zeta \in \eta} \cup\left\{0_{W \backslash V} \cup 1_{V}\right\}\right)$. Observe that the failure of the induction at stage $\eta$ is absolute between models with the same reals; in particular, the construction fails in $V$ at stage $\eta$ if it fails in $V[G]$ at that stage. So the remaining argument takes place in $V$.

Now, for $a \in 2, f \in \mathbb{P}(\kappa), q \in \mathbb{S}\left(\left\{s_{\zeta}\right\}_{\zeta \in \eta}\right)$ and $\beta \in \eta$, define a relation $\rho_{f, q, \beta}^{a} \subseteq$ $U^{a} \times \Phi^{*}\left(U^{a}\right)$ by letting $\rho_{f, q, \beta}^{a}(i, j)$ hold if and only if

$$
(f, q \cup\{(i, 0)\}) \Vdash_{\mathbb{P}\left(\left\{\bar{f}_{\eta}\right\}\right) \times \mathbb{S}\left(\left\{s_{\zeta}\right\}_{\zeta \in \eta}\right)} " j \notin \stackrel{\circ}{Y_{\beta}} "
$$

and

$$
(f, q \cup\{(i, 1)\}) \Vdash_{\mathbb{P}\left(\left\{\bar{f}_{\eta}\right\}\right) \times \mathbb{S}\left(\left\{s_{\zeta}\right\}_{\zeta \in \eta}\right)} " j \in \stackrel{\circ}{Y}_{\beta} " .
$$

It should be stressed here that the definition of $\rho_{f, q, \beta}^{a}$ depends only on those coordinates of $\mathbb{P}(\kappa)$ belonging to $\mathfrak{M}$ because coordinates of $f$ not belonging to $\mathfrak{M}$ have no influence on membership in $\stackrel{\circ}{Y}_{\beta}$. This remark will be crucial later when claiming that the function $B$, yet to be defined, is Borel.

Claim 1. If $a \in 2, X \in\left[U^{a}\right]^{\aleph_{0}}, h: X \rightarrow \omega$ and $f \Vdash_{\mathbb{P}(\kappa)}$ " $h$ induces $\stackrel{\circ}{\Phi}$ ", then there are $\bar{f} \supseteq f, q$, and $\beta$ such that $h \subseteq^{*} \rho_{\bar{f}, q, \beta}$.

Proof. This is essentially the argument from $(* 15)$ on page 189 of [6].

Claim 2. If $\bar{f} \supseteq f$, then $\rho_{\bar{f}, q, \beta}^{a} \supseteq \rho_{f, q, \beta}^{a}$ for any $a, q$ and $\beta$.

Proof. Suppose that $\rho_{f, q, \beta}^{a}(i, j)$ holds but $\rho_{\bar{f}, q, \beta}^{a}(i, j)$ fails. This means that either

$$
(\bar{f}, q \cup\{(i, 0)\}) \forall \forall_{\mathbb{P}\left(\left\{\bar{f}_{\eta}\right\}\right) \times \mathbb{S}\left(\left\{s_{\zeta}\right\}_{\zeta \in \eta}\right)} " j \notin \stackrel{\circ}{Y_{\beta}} "
$$

or

$$
(\bar{f}, q \cup\{(i, 1)\}) \forall \forall_{\mathbb{P}\left(\left\{\bar{f}_{\eta}\right\}\right) \times \mathbb{S}\left(\left\{s_{\zeta}\right\}_{\zeta \in \eta}\right)} " j \in \stackrel{\circ}{Y}_{\beta} "
$$


So assume the first possibility, the argument in the other case being similar. However, the dense sets in $\mathbb{P}_{\eta}$, that decide whether or not $j \notin \stackrel{\circ}{Y}_{\beta}$, remain dense in $\mathbb{P}\left(\left\{\bar{f}_{\eta}\right\}\right) \times \mathbb{S}\left(\left\{s_{\zeta}\right\}_{\zeta \in \eta}\right)$ by the choice of $\bar{f}_{\eta}$. Hence there is some $\left(f^{\prime}, q^{\prime}\right) \in \mathbb{P}_{\eta}$ such that

$$
\left(f^{\prime} \cup \bar{f}, q^{\prime} \cup q \cup\{(i, 0)\}\right) \in \mathbb{P}\left(\left\{\bar{f}_{\eta}\right\}\right) \times \mathbb{S}\left(\left\{s_{\zeta}\right\}_{\zeta \in \eta}\right)
$$

and $\left(f^{\prime}, q^{\prime}\right) \Vdash_{\mathbb{P}_{\eta}}$ " $j \in \stackrel{\circ}{Y}_{\beta}$ ". But then

$$
\left(f^{\prime} \cup f, q^{\prime} \cup q \cup\{(i, 0)\}\right) \in \mathbb{P}\left(\left\{\bar{f}_{\eta}\right\}\right) \times \mathbb{S}\left(\left\{s_{\zeta}\right\}_{\zeta \in \eta}\right)
$$

because $f \subseteq \bar{f}$ and this contradicts that

$$
(f, q \cup\{(i, 0)\}) \Vdash_{\mathbb{P}\left(\left\{\bar{f}_{\eta}\right\}\right) \times \mathbb{S}\left(\left\{s_{\zeta}\right\}_{\zeta \in \eta}\right)} " j \notin \stackrel{\circ}{Y}_{\beta} " .
$$

Claim 3. If $a \in 2, X \in\left[U^{a}\right]^{\aleph_{0}}, h: X \rightarrow \omega$ and $f \Vdash_{\mathbb{P}(\kappa)}$ " $h$ induces $\stackrel{\circ}{\Phi}$ ", then there exist $\bar{f}, q$ and $\beta$ such that $f \subseteq \bar{f}$ and $h \equiv^{*} \rho_{\bar{f}, q, \beta}^{a}\lceil X$.

Proof. Let $a \in 2$ be given and let $\left\{\left(q_{n}, \beta_{n}\right)\right\}_{n \in \omega}$ enumerate $\mathbb{S}\left(\left\{s_{\zeta}\right\}_{\zeta \in \eta}\right) \times \eta$ infinitely often. Let $f_{0}=f$ and construct $f_{n}$ inductively as follows. Given $f_{m} \in \mathbb{P}(\kappa)$ and a finite subset $A_{m}$ of the support of $f_{m}$, choose an integer $k_{m}$ large enough that for each $\alpha \in A_{m}$ there is some $k \in k_{m}$ such that $\mid\left[2^{k}, 2^{k+1}\right) \backslash$ domain $\left(f_{m}(\alpha)\right) \mid \geq m$. Let $\left\{g_{i}\right\}_{i=0}^{L}$ enumerate all $g \in \mathbb{P}\left\langle A_{m}, k_{m}\right\rangle$ such that $g \cup f_{m} \in \mathbb{P}(\kappa)$. Let $f_{m, 0}=f_{m}$ and choose inductively, if possible, $f_{m, i+1}^{\prime} \supseteq f_{m, i}$ such that $f_{m, i+1}^{\prime}\left\langle A_{m}, k_{m}\right\rangle=$ $f_{m, i}\left\langle A_{m}, k_{m}\right\rangle$ and $h \subseteq \rho_{f_{m, i+1}^{\prime}}^{a} \cup g_{i}, q_{m}, \beta_{m}$. There are three possibilities to consider.

Case One. The first case to consider is that it is not possible to find $f_{m, i+1}^{\prime}$ such that $f_{m, i+1}^{\prime} \supseteq f_{m, i}$ and $f_{m, i+1}^{\prime}\left\langle A_{m}, k_{m}\right\rangle=f_{m, i}\left\langle A_{m}, k_{m}\right\rangle$ and $h \subseteq \rho_{f_{m, i+1}^{\prime} \cup g_{i}, q_{m}, \beta_{m}}^{a}$. In this case let $f_{m, i+1}=f_{m, i}$.

Case Two. If $f_{m, i+1}^{\prime}$ is defined and $h \equiv^{*} \rho_{f_{m, i+1}^{\prime} \cup g_{i}, q_{m}, \beta_{m}}^{a} \uparrow X$, then let $\bar{f}=$ $f_{m, i+1}^{\prime} \cup g_{i}, q=q_{m}$ and $\beta=\beta_{m}$. This satisfies the conclusion of Claim 3

Case Three. Otherwise, the range of $\rho_{f_{m, i+1}^{\prime} \cup g_{i}, q_{m}, \beta_{m}}^{a} \backslash h$ contains an infinite set $Z$. The definition of $\rho_{f_{m, i+1}^{\prime} \cup g_{i}, q_{m}, \beta_{m}}^{a}$ and Corollary 3.3 imply that there are $W_{m, i} \in$ $\left[U^{a}\right]^{\aleph_{0}}$ and $f_{m, i+1} \supseteq f_{m, i+1}^{\prime}$ such that

$$
f_{m, i+1} \Vdash_{\mathbb{P}(\kappa)} \text { " } \stackrel{\circ}{\Phi}^{*}\left(W_{m, i}\right) \subseteq^{*} Z \text { and } W_{m, i} \in \mathcal{T}(\stackrel{\circ}{\Phi}) "
$$

and $f_{m, i+1}\left\langle A_{m}, k_{m}\right\rangle=f_{m, i}\left\langle A_{m}, k_{m}\right\rangle$. Notice that the last condition is easily satisfied, since forcing with $\mathbb{P}$ is stable under the finite modification of finitely many coordinates.

Now let $f_{m+1}=f_{m, L}$ and let $f_{\omega}=\bigcup_{m \in \omega} f_{m}$. If the sets $A_{m}$ have been chosen wisely, the construction guarantees that $f_{\omega} \in \mathbb{P}(\kappa)$ and $f_{m, i} \leq_{\kappa}^{*} f_{\omega}$ for all $m$ and $i$. Hence, from Corollary 3.3, it follows that there is some $W \subseteq \omega$ and $\tilde{f} \supseteq f_{\omega}$ such that $W \supseteq X$ and $W \supseteq{ }^{*} W_{m, i}$ for each relevant $m$ and $i$ and, furthermore, there is some one-to-one function $\bar{h}: W \rightarrow \omega$ such that $\tilde{f} \Vdash_{\mathbb{P}(\kappa)}$ " $\bar{h}$ induces $\stackrel{\circ}{\Phi}$ on $W$ ". From Claim[1it follows that there are $\bar{f} \supseteq \tilde{f}$ and $m$ such that $\bar{h} \subseteq^{*} \rho_{\bar{f}, q_{m}, \beta_{m}}^{a}$. Since $X \subseteq W$, it follows that $h \subseteq^{*} \bar{h}$ and, hence, $h \subseteq^{*} \rho_{\bar{f}, q_{m}, \beta_{m}}^{a}$. 
If $h \not *^{*} \rho_{\bar{f}, q_{m}, \beta_{m}}^{a} \uparrow X$, then it must be that there is some infinite $Z$ contained in the range of $\left(\rho_{\bar{f}, q_{m}, \beta_{m}}^{a} \uparrow X\right) \backslash h$. Let $i$ be such that $g_{i} \supseteq \bar{f}\left\langle A_{m}, k_{m}\right\rangle$. It must be that Case 3 holds in the construction of $f_{m, i+1}$, since $\bar{f}$ witnesses that Case 1 could not hold and, if Case 2 were in force, then the claim would already have been established. Therefore $W_{m, i} \subseteq^{*} W$. Using that $X \cap \bar{W}$ is finite, choose $j_{1} \in X \backslash \operatorname{domain}\left(q_{m}\right)$ and $k$ belonging to the range of $\rho_{\bar{f}, q_{m}, \beta_{m}}^{a} \cap \bar{h} \uparrow W_{m, i}$ such that $\rho_{f_{m, i+1} \cup g_{i}, q_{m}, \beta_{m}}^{a}\left(j_{1}, k\right)$ holds, $h\left(j_{1}\right) \neq k$ and $h\left(j_{1}\right)=\bar{h}\left(j_{1}\right)$. Let $j_{2}$ be such that $\bar{h}\left(j_{2}\right)=k$. Then $j_{1} \neq j_{2}$ and $\rho_{\bar{f}, q_{m}, \beta_{m}}^{a}\left(j_{1}, k\right)$ holds from Claim 2 because $\bar{f} \supseteq f_{\omega} \cup g_{i} \supseteq f_{m, i+1} \cup g_{i}$. Moreover,

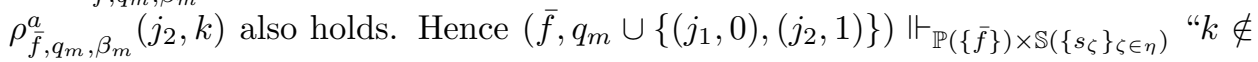
$\stackrel{\circ}{Y}_{\beta}$ and $k \in \stackrel{\circ}{Y}_{\beta}$ ". This contradiction means that Case 2 must have held at some point in the construction.

Claim 4. If $a \in 2$ and $f \in \mathbb{P}(\kappa)$, then there exist $f^{*} \supseteq f, q$ and $\beta$ such that for any $X \in\left[U^{a}\right]^{\aleph_{0}}, h: X \rightarrow \omega$ and $\tilde{f} \supseteq f^{*}$ such that

$$
\tilde{f} \vdash_{\mathbb{P}(\kappa)} \text { " } h \text { induces } \Phi \text { on } X ",
$$

there exist $\bar{f} \supseteq \tilde{f}$ such that $h \equiv{ }^{*} \rho_{\bar{f}, q, \beta}^{a} \uparrow X$.

Proof. Let $a \in 2$ be given. Let $\left\{\left(q_{n}, \beta_{n}\right)\right\}_{n \in \omega}$ enumerate $\mathbb{S}\left(\left\{s_{\zeta}\right\}_{\zeta \in \eta}\right) \times \eta$. Let $f_{0}=f$ and construct $f_{n}$ inductively as follows. Given $f_{m} \in \mathbb{P}(\kappa)$ and a finite subset $A_{m}$ of the support of $f_{m}$, choose an integer $k_{m}$ large enough that for each $\alpha \in A_{m}$ there is some $k \in k_{m}$ such that $\mid\left[2^{k}, 2^{k+1}\right) \backslash$ domain $\left(f_{m}(\alpha)\right) \mid \geq m$. Let $\left\{g_{i}\right\}_{i=0}^{L}$ enumerate all $g \in \mathbb{P}\left\langle A_{m}, k_{m}\right\rangle$ such that $g \cup f_{m} \in \mathbb{P}(\kappa)$. Let $f_{m, 0}=f_{m}$ and choose inductively, if possible, $f_{m, i+1} \supseteq f_{m, i}$ such that $f_{m, i+1}\left\langle A_{m}, k_{m}\right\rangle=f_{m, i}\left\langle A_{m}, k_{m}\right\rangle$ and, moreover, there is some $h_{m, i}: X_{m, i} \rightarrow \omega$ such that $X_{m, i} \subseteq U^{a}$ and

$$
f_{m, i+1} \Vdash_{\mathbb{P}(\kappa)} \text { " } h_{m, i} \text { induces } \stackrel{\circ}{\Phi} \text { on } X_{m, i} \text { " }
$$

and $h_{m, i} \not \equiv^{*} \rho_{f^{\prime}, q_{m}, \beta_{m}}^{a} \uparrow X_{m, i}$ for all $f^{\prime} \supseteq f_{m, i+1} \cup g_{i}$. If this fails, then setting $f^{*}=f_{m, i} \cup g_{i}, q \stackrel{q}{=} q_{m}$ and $\beta=\beta_{m}$ satisfies the claim. Hence suppose that the construction never fails and let $f_{m+1}=f_{m, L}$ and define $f_{\omega}=\bigcup_{m \in \omega} f_{m}$. As in the proof of Claim 3 it is possible to find $\tilde{f} \supseteq f_{\omega}$ and $\tilde{h}: X \rightarrow \omega$ such that $U^{a} \supseteq X \supseteq^{*} X_{m, i}$ for all $m$ and $i$ and such that $\tilde{f} \Vdash_{\mathbb{P}(\kappa)}$ " $\tilde{h}$ induces $\stackrel{\circ}{\Phi}$ on $X$ ". Now use Claim 3 to find $\bar{f} \supseteq \tilde{f}$ and $m$ such that $\tilde{h} \equiv{ }^{*} \rho_{\bar{f}, q_{m}, \beta_{m}}$. Let $i$ be such that $\bar{f}\left\langle A_{m}, k_{m}\right\rangle \subseteq g_{i}$ and note that $\bar{f} \cup g_{i} \supseteq f_{m, i+1} \cup g_{i}$ and $h_{m, i} \equiv^{*} \rho_{\bar{f} \cup g_{i}, q_{m}, \beta_{m}}\left\lceil X_{m, i}\right.$. This contradicts the construction hypothesis.

$$
\begin{aligned}
& \text { Now, for any }\left(q_{0}, q_{1}\right) \in \mathbb{S}\left(\left\{s_{\zeta}\right\}_{\zeta \in \eta}\right)^{2} \text { and }\left(\beta_{0}, \beta_{1}\right) \in \eta^{2} \text { define } B_{q_{0}, q_{1}, \beta_{0}, \beta_{1}} \text { by }
\end{aligned}
$$

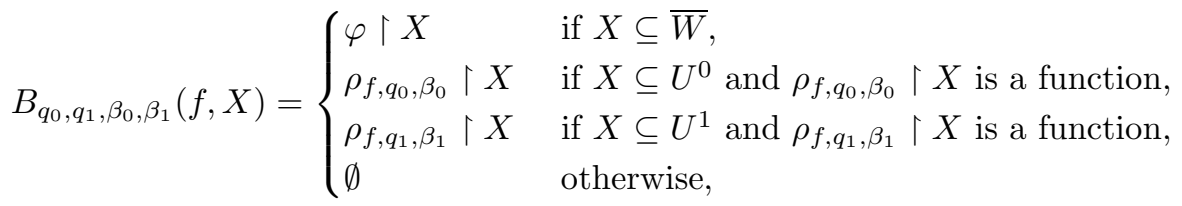

where $f \in \mathbb{P}(\kappa)$ and $X \in[\omega]^{\aleph_{0}}$. Notice that $B_{q_{0}, q_{1}, \beta_{0}, \beta_{1}}$ is a Borel function. It will be shown that the set of conditions $f \in \mathbb{P}(\kappa)$ such that there are $q_{0}, q_{1}, \beta_{0}$ and $\beta_{1}$ satisfying

$$
f \Vdash_{\mathbb{P}(\kappa)} \text { " } B_{q_{0}, q_{1}, \beta_{0}, \beta_{1}} \text { satisfies the conclusion of Theorem } 4.1 \text { ' }
$$

is dense. 
To this end, let $f \in \mathbb{P}(\kappa)$ be arbitrary. Apply Claim 4 to $f$ and $a=0$ to find $f^{0} \supseteq f, q^{0}$ and $\beta^{0}$ satisfying the conclusion of Claim 4 Then use Claim 4 again, but now applied to $f^{0}$ and $a=1$ to find $f^{1} \supseteq f^{0}, q^{1}$ and $\beta^{1}$. Claim 4 together with the genericity of $F$ over $V$ guarantees that if $f^{1} \in F$, then, in $V[F]$, for every $a \in 2$ and $X \in\left[U^{a}\right]^{\aleph_{0}}$ such that $h$ induces $\Phi$ on $X$, there is some $\bar{f} \in F$ such that

$$
h \equiv^{*} \rho_{\bar{f}, q^{a}, \beta^{a}} \uparrow X
$$

or, in other words, $B_{q^{0}, q^{1}, \beta^{0}, \beta^{1}}(\bar{f}, X)$ induces $\Phi$ on $X$. This amounts to the same as saying that $f^{1}$ forces that the Borel relation $B_{q^{0}, q^{1}, \beta^{0}, \beta^{1}}$ satisfies the conclusion of the theorem.

The final, "moreover", clause is immediate from the definition of $B_{q^{0}, q^{1}, \beta^{0}, \beta^{1}}$. To see this, suppose that $B_{q^{0}, q^{1}, \beta^{0}, \beta^{0}}(f, X)$ and $B_{q^{0}, q^{1}, \beta^{0}, \beta^{1}}\left(f^{\prime}, X^{\prime}\right)$ are nonempty and $X \subseteq X^{\prime}$ and $f \supseteq f^{\prime}$. If $X^{\prime} \subseteq \bar{W}$, then the result is clear; so let $a$ be such that $X^{\prime} \subseteq U_{a}$. Then Claim 2 implies that

$$
\rho_{f, q^{a}, \beta^{a}} \supseteq \rho_{f^{\prime}, q^{a}, \beta^{a}} .
$$

Since both $B_{q^{0}, q^{1}, \beta^{0}, \beta^{0}}(f, X)$ and $B_{q^{0}, q^{1}, \beta^{0}, \beta^{1}}\left(f^{\prime}, X^{\prime}\right)$ are nonempty, it follows that $\rho_{f, q^{a}, \beta^{a}}\left\lceil X\right.$ and $\rho_{f^{\prime}, q^{a}, \beta^{a}} \uparrow X^{\prime}$ are both functions and, since $X \subseteq X^{\prime}$, so is $\rho_{f^{\prime}, q^{a}, \beta^{a}} \uparrow$ $X$. From (4.5) it follows that

$$
\rho_{f, q^{a}, \beta^{a}} \uparrow X=\rho_{f^{\prime}, q^{a}, \beta^{a}} \uparrow X
$$

and hence $B_{q^{0}, q^{1}, \beta^{0}, \beta^{1}}(f, X) \subseteq B_{q^{0}, q^{1}, \beta^{0}, \beta^{1}}\left(f^{\prime}, X^{\prime}\right)$.

Corollary 4.1. In $V[F]$ the number of automorphisms of $\mathcal{P}(\omega) /[\omega]^{<\aleph_{0}}$ is between $\kappa$ and $\kappa^{\aleph_{0}}$.

Proof. From Lemma 3.3 it follows that it suffices to show that there are no more than $\kappa^{\aleph_{0}}$ nontrivial automorphisms of $\mathcal{P}(\omega) /[\omega]^{<\aleph_{0}}$. If there are, then it follows from Theorem 4.1 that there are two automorphisms $\Phi$ and $\Psi$ and there is a single Borel function $B$ such that the following hold:

$$
\begin{gathered}
\left(\forall W \in[\omega]^{\aleph_{0}}\right)(\forall f \in F)\left(\exists \bar{W} \in[W]^{\aleph_{0}}\right)(\exists \bar{f} \in F) \bar{f} \supseteq f \text { and } \\
\bar{f} \Vdash_{\mathbb{P}(\kappa)} \text { " }(\forall Z \subseteq \bar{W}) \stackrel{\circ}{*}(Z) \Phi^{*} B(f, \bar{W})(Z) ", \\
\left(\forall W \in[\omega]^{\aleph_{0}}\right)(\forall f \in F)\left(\exists \bar{W} \in[W]^{\aleph_{0}}\right)(\exists \bar{f} \in F) \bar{f} \supseteq f \text { and } \\
\bar{f} \Vdash_{\mathbb{P}(\kappa)} \text { " }(\forall Z \subseteq \bar{W}) \Psi^{*}(Z) \equiv^{*} B(f, \bar{W})(Z) " .
\end{gathered}
$$

Using the fact that $\Psi$ and $\Phi$ are forced to be different, it is possible to find $W \in$ $[\omega]^{\aleph_{0}}$ and $f^{\prime} \in F$ such that $f^{\prime} \Vdash_{\mathbb{P}(\kappa)}$ " $\left|\Phi^{*}(W) \cap \stackrel{\circ}{*}(W)\right|<\aleph_{0}^{*}$. From (4.6) it follows that there is $f_{1} \in F$ such that $f_{1} \supseteq f^{\prime}$ and $X \subseteq W$ such that $f_{1} \Vdash_{\mathbb{P}(\kappa)}$ " $B\left(f_{1}, X\right)$ induces $\stackrel{\circ}{\Phi}$ on $X$ ". From (4.7), it follows that there is some $f_{2} \in F$ such that $f_{2} \supseteq f_{1}$ and there is $\bar{X} \subseteq X$ such that $f_{2} \Vdash_{\mathbb{P}(\kappa)}$ " $B\left(f_{2}, \bar{X}\right)$ induces $\stackrel{\circ}{\Psi}$ on $\bar{X}$ ". But now, applying the "moreover" clause of Theorem 4.1, it follows that $B\left(f_{2}, \bar{X}\right) \subseteq B\left(f_{1}, X\right)$, since both are nonempty. Hence

$$
f_{2} \Vdash_{\mathbb{P}} \text { “ } \stackrel{\circ}{ }^{*}(\bar{X}) \equiv \equiv^{*} B\left(f_{1}, X\right)(\bar{X}) \equiv \equiv^{*} B\left(f_{2}, \bar{X}\right)(\bar{X}) \equiv \stackrel{\circ}{\Psi^{*}}(\bar{X}) ",
$$

contradicting that $\bar{X} \subseteq W$. 
Corollary 4.2. It is consistent that $2^{\aleph_{0}}=\aleph_{2}, 2^{2^{\aleph_{0}}}=\aleph_{4}$ and the number of automorphisms of $\mathcal{P}(\omega) /[\omega]^{<\aleph_{0}}$ is exactly $\aleph_{3}$.

Proof. Start with a model $V$ of PFA where $2^{\aleph_{2}}=\aleph_{4}$ and then force with $\mathbb{P}\left(\aleph_{3}\right)$. Since $\aleph_{3}^{\aleph_{0}}=\aleph_{3}$ in the ground model, it follows from Corollary 4.2 that there are exactly $\aleph_{3}$ automorphisms of $\mathcal{P}(\omega) /[\omega]^{<\aleph_{0}}$ in the generic extension. Since no new reals are added, the continuum does not change and because the forcing has size $\aleph_{3}, \aleph_{4}$ is not collapsed.

\section{Comments}

The automorphism of $\mathcal{P}(\omega) /[\omega]<\aleph_{0}$ added by $\mathbb{P}$ is trivial not only on a dense $P$-ideal, but on a dense $P_{\aleph_{1}}$-ideal. This raises the following question.

Question 5.1. In $V[F]$ is every automorphism trivial on a $P_{\aleph_{1}}$ - ideal?

The next result is intended as motivation for the next question. Recall the definition of $\Phi_{G}$ in Definition 3.3 where $G$ is a filter on $\mathbb{P}$.

Definition 5.1. Given a tower $\mathcal{F}=\left\{f_{\xi}\right\}_{\xi \in \kappa} \subseteq \mathbb{P}$, define $\mathfrak{C}_{\xi}$ to be the set of all $a \in\left[\operatorname{domain}\left(f_{\xi}\right)\right]^{<\aleph_{0}}$ such that $f_{\xi}\lceil a$ is a cycle. Let

$$
f_{\xi}^{*}(n)= \begin{cases}f_{\xi}(n) & \text { if } n \in a \in \mathfrak{C}_{\xi} \text { and }|a| \text { is odd, } \\ n & \text { otherwise. }\end{cases}
$$

Proposition 5.1. If a tower $\mathcal{F}=\left\{f_{\xi}\right\}_{\xi \in \kappa} \subseteq \mathbb{P}$ is obtained generically, then the tower of functions $\mathcal{F}^{*}=\left\{f_{\xi}^{*}\right\}_{\xi \in \kappa}$ as defined in Definition 5.1 is such that

$$
\Phi_{\mathcal{F}^{*}} \neq \Phi_{\mathcal{F}}^{n_{0}} g_{1} \Phi_{\mathcal{F}}^{n_{1}} g_{2} \Phi_{\mathcal{F}}^{n_{2}} \ldots g_{k} \Phi_{\mathcal{F}}^{n_{k}}
$$

for any choice of $\left\{n_{i}\right\}_{i=0}^{k}$ and almost permutations $\left\{g_{i}\right\}_{i=1}^{k}$. In other words, $\Phi_{\mathcal{F} *}$ does not belong to the subgroup generated by $\Phi_{\mathcal{F}}$ and the trivial automorphisms.

Proof. Let $f \in \mathbb{P}$ and suppose that $\left\{n_{i}\right\}_{i=0}^{k}$ and $\left\{g_{i}\right\}_{i=1}^{k}$ are given. Choose $X \subseteq \omega$ such that $\left[2^{x}, 2^{x+1}\right) \backslash \operatorname{domain}(f)$ has at least $(3 k)^{k} \prod_{i=0}^{k} n_{i}$ elements for each $x \in X$ and, moreover, the set

$$
\left\{\left|\left[2^{x}, 2^{x+1}\right) \backslash \operatorname{domain}(f)\right|: x \in \omega \backslash X\right\}
$$

is unbounded. For each $x \in X$ choose $j_{x} \in\left[2^{x}, 2^{x+1}\right) \backslash$ domain $(f)$.

For any $j \in \omega$ let $\beta(j)$ be the set of all $g_{i}^{\epsilon_{1}} g_{i+1}^{\epsilon_{2}}, \ldots, g_{i+m}^{\epsilon_{m}}(j)$ such that $1 \leq i$, $m \leq k-i$ and $\left(\epsilon_{1}, \epsilon_{2}, \ldots, \epsilon_{m}\right) \in\{-1,1\}^{m}$. For a set $a \subseteq \omega$, let $\bar{\beta}(a)=\bigcup_{j \in a} \beta(j)$. Now define an increasing sequence of functions $f_{x}^{n}$ and integers $j_{x}^{n}$ by induction, starting with $f_{x}^{0}=\emptyset$ and $j_{x}^{0}=j_{x}$. Let $N=\sum_{i \in k}\left|n_{i}\right|+k$. If $n \leq N$ and $n \notin\left\{\sum_{i \in m}\left|n_{i}\right|+m: m \leq k\right\}$, then there are three cases to consider. If $f_{x}^{n}\left(j_{x}^{n}\right)$ is defined, then let $f_{x}^{n}=f_{x}^{n+1}$ and $j_{x}^{n+1}=f_{x}^{n}\left(j_{x}^{n}\right)$. Otherwise, choose $j_{x}^{n+1}$ in $\left[k_{x}, k_{x+1}\right) \backslash\left(\operatorname{domain}(f) \cup \bar{\beta}\left(\left\{j_{x}^{i}\right\}_{i=0}^{n}\right)\right)$. Then, if $\sum_{i \in m}\left|n_{i}\right|+m<n \leq n_{m}$ and $n_{m}>0$, define $f_{x}^{n+1}=f_{x}^{n} \cup\left\{\left(j_{x}^{n}, j_{x}^{n+1}\right)\right\}$ while, if $n_{m}<0$, define $f_{x}^{n+1}=f_{x}^{n} \cup\left\{\left(j_{x}^{n+1}, j_{x}^{n}\right)\right\}$. If $n=\sum_{i \in m}\left|n_{i}\right|+m$ for some $m$, then define $f_{x}^{n+1}=f_{x}^{n}$ and $j_{x}^{n+1}=g_{m}\left(j_{x}^{n}\right)$.

Observe that $f_{x}^{N}$ is one-to-one. Moreover, if $m$ is such that $g_{m}\left(j_{x}^{n}\right) \neq j_{x}^{n}$ where $n=\sum_{i \in m}\left|n_{i}\right|+m$, then it is possible to extend $f_{x}^{N}$ to two cycles, one containing $j_{x}$ and the other containing

$$
\Phi_{\mathcal{F}}^{n_{0}} g_{1} \Phi_{\mathcal{F}}^{n_{1}} g_{2} \Phi_{\mathcal{F}}^{n_{2}} \ldots g_{k} \Phi_{\mathcal{F}}^{n_{k}}\left(j_{x}\right) .
$$


On the other hand, if $g_{m}\left(j_{x}^{n}\right)=j_{x}^{n}$ for all $n$ such that $n=\sum_{i \in m}\left|n_{i}\right|+m$, then it is possible to extend $f_{x}^{N}$ to an even permutation if $\sum_{i \in k} n_{i} \neq 0$ and to an odd cycle if $\sum_{i \in k} n_{i}=0$. Let $f^{\prime}=f \cup \bigcup_{x \in X} f_{x}^{N}$ and note that $f^{\prime}$ forces that

$$
\Phi_{\mathcal{F}}^{n_{0}} g_{1} \Phi_{\mathcal{F}}^{n_{1}} g_{2} \Phi_{\mathcal{F}}^{n_{2}} \ldots g_{k} \Phi_{\mathcal{F}}^{n_{k}}\left(\left\{j_{x}: x \in X\right\}\right) \not^{*} \Phi_{\mathcal{F}^{*}}\left(\left\{j_{x}: x \in X\right\}\right) .
$$

Question 5.2. Is it consistent that there is a nontrivial automorphism $\Psi$ of $\mathcal{P}(\omega) /[\omega]<\aleph_{0}$ yet every automorphism of $\mathcal{P}(\omega) /[\omega]<\aleph^{<}$is algebraically generated by the $\Psi$ together with the trivial automorphisms? Proposition 5.1 shows that this is not so for the automorphisms and models discussed in this paper.

The gap between the two cardinals mentioned as possibilities in Corollary 4.1 points to the next question.

Question 5.3. Is it consistent that the cardinality of the automorphism group of $\mathcal{P}(\omega) /[\omega]<\aleph_{0}$ has countable cofinality?

One might try to disprove this by assuming that the set of all automorphisms of $\mathcal{P}(\omega) /[\omega]<\aleph_{0}$ has cardinality $\aleph_{\lambda}$, where $\lambda$ has countable cofinality, and then choosing a partition of the integers into infinite sets $\left\{A_{n}\right\}_{n \in \omega}$ and letting the set of all automorphisms of $\mathcal{P}(\omega) /[\omega]<\aleph_{0}$ be expressed as $\bigcup_{n \in \omega} \mathfrak{A}_{n}$ such that $\left|\mathfrak{A}_{n}\right|<\aleph_{\lambda}$. It is then possible to find automorphisms $\Phi_{n}$ of $\mathcal{P}\left(A_{n}\right) /\left[A_{n}\right]<\aleph_{0}$ that disagree with every member of $\mathfrak{A}_{n}$. If these could be glued together somehow, a contradiction would soon be in hand. A solution of the "glueing" problem is likely to have other applications as well.

\section{REFERENCES}

[1] Uri Abraham, Matatyahu Rubin, and Saharon Shelah, On the consistency of some partition theorems for continuous colorings, and the structure of $\aleph_{1}$-dense real order types, Ann. Pure Appl. Logic, 29(2):123-206, 1985. MR 87d:03132

[2] Winfried Just, A modification of Shelah's oracle-c.c. with applications, Trans. Amer. Math. Soc., 329(1):325-356, 1992. MR 92j:03047

[3] S. Shelah, Proper Forcing, Lecture Notes in Mathematics, vol. 940, Springer-Verlag, Berlin, 1982. MR 84h:03002

[4] S. Shelah and J. Steprāns, PFA implies all automorphism are trivial, Proc. Amer. Math. Soc., 104:1220-1225, 1988. MR 89e:03080

[5] S. Shelah and J. Steprāns, Somewhere trivial autohomeomorphisms, J. London Math. Soc. (2), 49:569-580, 1994. MR 95f:54008

[6] Saharon Shelah, Proper and improper forcing, Perspectives in Mathematical Logic, SpringerVerlag, Berlin, second edition, 1998. MR 98m:03002

[7] S. Todorcevic, Partition Problems in Topology, Contemporary Mathematics, vol. 84, American Mathematical Society, Providence, RI, 1989. MR 90d:04001

[8] Boban Veličković, Definable automorphisms of $\mathcal{P}(\omega) /$ fin, Proc. Amer. Math. Soc., 96(1):130135, 1986. MR 87 m:03070

[9] Boban Veličković, OCA and automorphisms of $\mathcal{P}(\omega) /$ fin, Topology Appl., 49(1):1-13, 1993. MR 94a:03080

Department of Mathematics, York University, 4700 Keele Street, North York, OnTARIO, CANADA M3J 1P3

E-mail address: steprans@yorku.ca 